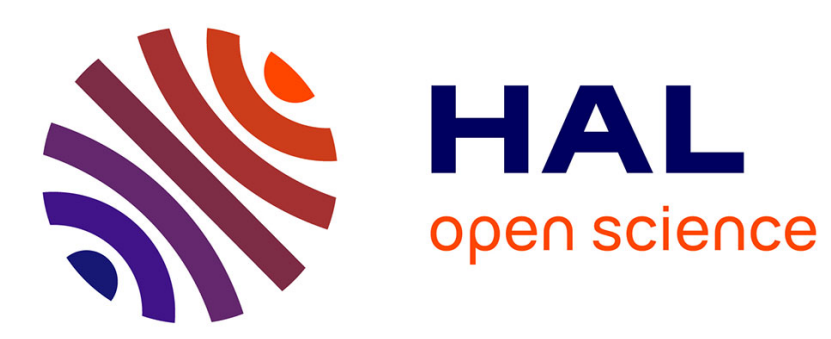

\title{
On Some Applications of Generalized Functionality for Arithmetic Discrete Planes
}

\author{
Valerie Berthe, Christophe Fiorio, Damien Jamet, Fabrice Philippe
}

\section{To cite this version:}

Valerie Berthe, Christophe Fiorio, Damien Jamet, Fabrice Philippe. On Some Applications of Generalized Functionality for Arithmetic Discrete Planes. Image and Vision Computing, 2007, 25 (10), pp.1671-1684. 10.1016/j.imavis.2006.06.023 . lirmm-00182676

\section{HAL Id: lirmm-00182676 https://hal-lirmm.ccsd.cnrs.fr/lirmm-00182676}

Submitted on 7 Jun 2011

HAL is a multi-disciplinary open access archive for the deposit and dissemination of scientific research documents, whether they are published or not. The documents may come from teaching and research institutions in France or abroad, or from public or private research centers.
L'archive ouverte pluridisciplinaire HAL, est destinée au dépôt et à la diffusion de documents scientifiques de niveau recherche, publiés ou non, émanant des établissements d'enseignement et de recherche français ou étrangers, des laboratoires publics ou privés. 


\title{
On some Applications of Generalized Functionality for Arithmetic Discrete Planes
}

\author{
Valérie Berthé, Christophe Fiorio, Damien Jamet, Fabrice Philippe \\ LIRMM, UMR 5506, Univ. Montpellier II, 161 rue Ada, F-34392 Montpellier \\ Cedex 05, France
}

\begin{abstract}
Naive discrete planes are well known to be functional on a coordinate plane. The aim of our paper is to extend the functionality concept to a larger family of arithmetic discrete planes, by introducing suitable projection directions $\left(\alpha_{1}, \alpha_{2}, \alpha_{3}\right)$ satisfying $\alpha_{1} v_{1}+\alpha_{2} v_{2}+\alpha_{3} v_{3}=w$. Several applications are considered. We first study certain local configurations, that is, the $(m, n)$-cubes introduced in [VC99]. We compute their number for a given $(m, n)$ and study their statistical behaviour. We then apply functionality to formulate an algorithm for generating arithmetic discrete planes, inspired by [Deb95]. We also prove that an arithmetic discrete plane may be endowed with a combinatorial surface structure, in the spirit of [KI03].
\end{abstract}

Key words: digital planes; arithmetic planes; local configurations; functionality of discrete planes.

\section{Introduction}

Let $\mathbf{v}=\left(v_{1}, v_{2}, v_{3}\right) \in \mathbb{Z}^{3}$ and $\mu, w \in \mathbb{Z}^{2}$. The arithmetic discrete plane $\mathfrak{P}(\mathbf{v}, \mu, w)$ is the set of all points $\mathbf{x}=\left(x_{1}, x_{2}, x_{3}\right) \in \mathbb{Z}^{3}$ satisfying

$$
0 \leq v_{1} x_{1}+v_{2} x_{2}+v_{3} x_{3}+\mu<w
$$

Arithmetic discrete planes with a common normal vector $\mathbf{v}$ are mainly characterized by their thickness $w$. For example, in such a class the naive planes $\left(w=\max \left(\left|v_{1}\right|,\left|v_{2}\right|,\left|v_{3}\right|\right)\right)$ are the thinest 2-separating ones, while the standard planes $\left(w=\left|v_{1}\right|+\left|v_{2}\right|+\left|v_{3}\right|\right)$ are the thinest 0-separating ones (see [AAS97]).

Email addresses: berthe@lirmm.fr (Valérie Berthé), fiorio@lirmm.fr (Christophe Fiorio), jamet@lirmm.fr (Damien Jamet), philippe@lirmm.fr (Fabrice Philippe). 
Arithmetic discrete planes play a key role in polyedrisation of discrete objects [Deb95,BAB02,BK04]. Indeed tridimensional discretized objects are usually described as pieces, generally triangular, of arithmetic discrete planes. A reasonable polyedrisation mainly requires that the involved objects have good topological properties, such as connectedness and/or absence of $\kappa$-tunnels, for $\kappa \in\{0,1,2\}$ [AAS97] (intuitively, a $\kappa$-tunnel occurs if two voxels, one above the plane and the other below, have a $k$-dimensional intersection). If naive arithmetic planes are rather good candidates for this approach as being the thinnest ones without 2-tunnels, one major withdraw is that the intersection of two naive arithmetic discrete planes is usually not an arithmetic discrete line. Similar problems occur when considering standard arithmetic discrete planes. V. Brimkov and R. Barneva introduced a new class of arithmetic discrete planes [BB99,BB02], the so-called graceful planes, defined as $\mathfrak{P}(\mathbf{v}, \mu, w)$ with $0 \leq v_{1} \leq v_{2} \leq v_{3}$ and $w=\max \left\{v_{3}, v_{1}+v_{2}\right\}$, which allow for constructing thin discrete triangular tunnel-free meshes admitting analytical description. In order to take into account noise during digitization, I. Debled-Rennesson, J.-L. Rémy, and J. Rouyer-Degli initiated a new approach of segmentation of discrete curves with arithmetic discrete lines of various thicknesses [DRR03]. The latter works indicate that a better understanding of the topological, arithmetic and geometric structure of arithmetic discrete linear objects of any thickness would be very useful.

Naive planes have been widely studied (see for instance [Rev91,DR94,Deb95], [AAS97], [VC97], [BBN00a,BBN00b,BB02], and [Col02]) and are well known to be functional, that is, in a one-to-one correspondence with the integer points of one of the coordinate planes by an orthogonal projection map. In other words, given a naive arithmetic discrete plane $\mathfrak{P}$ and the suitable coordinate plane, for any integer point $P$ of this coordinate plane there exists a unique point of $\mathfrak{P}$ obtained by adding a third coordinate to $P$.

The aim of the present paper is to extend the notion of functionality for naive arithmetic discrete planes to a larger family of arithmetic discrete planes. For that purpose, instead of projecting on a coordinate plane, we introduce a suitable orthogonal projection map on a plane along a direction $\boldsymbol{\alpha}=\left(\alpha_{1}, \alpha_{2}, \alpha_{3}\right) \in$ $\mathbb{Z}^{3}$, in some sense dual to the normal vector of the discrete plane $\mathfrak{P}(\mathbf{v}, \mu, w)$, that is, $\alpha_{1} v_{1}+\alpha_{2} v_{2}+\alpha_{3} v_{3}=w$, so that the projection of $\mathbb{Z}^{3}$ and the points of the discrete plane $\mathfrak{P}(\mathbf{v}, \mu, w)$ are in one-to-one correspondence. Functionality allows us to reduce a three-dimensional problem to a two-dimensional one, thus leads to a better understanding of the combinatorial and geometric properties of arithmetic discrete planes.

The present paper is organized as follows. We first recall some basic notions on arithmetic discrete planes, while extending their definition to the case of real $(\mathbf{v}, \mu, w)$-parameters (we call rational the classic case).

Generalized functionality is introduced in Section 3, with the main result: 
Theorem 1 Let $\mathfrak{P}(\mathbf{v}, \mu, w)$ be an arithmetic discrete plane, and let $\boldsymbol{\alpha} \in \mathbb{Z}^{3}$ be such that $\operatorname{gcd}\left\{\alpha_{1}, \alpha_{2}, \alpha_{3}\right\}=1$. Let $\pi_{\boldsymbol{\alpha}}^{\perp}: \mathbb{R}^{3} \longrightarrow\left\{\mathbf{x} \in \mathbb{R}^{3},\langle\boldsymbol{\alpha}, \mathbf{x}\rangle=0\right\}$ be the affine orthogonal projection map onto the plane $\left\{\mathbf{x} \in \mathbb{R}^{3},\langle\boldsymbol{\alpha}, \mathbf{x}\rangle=0\right\}$ along the vector $\boldsymbol{\alpha}$. Then the map $\pi_{\boldsymbol{\alpha}}^{\perp}: \mathfrak{P}(\mathbf{v}, \mu, w) \longrightarrow \pi_{\boldsymbol{\alpha}}^{\perp}\left(\mathbb{Z}^{3}\right)$ is a bijection if, and only if, $|\langle\boldsymbol{\alpha}, \mathbf{v}\rangle|=w$.

Up to the sign, such $\boldsymbol{\alpha}$-vectors are called functional for the plane. We show that any rational arithmetic discrete plane admits functional vectors.

In Section 4, we study the functional lattice $\Gamma_{\boldsymbol{\alpha}}$ obtained by projecting an arithmetic discrete plane on one of the coordinate planes along a functional vector $\boldsymbol{\alpha}$, and we compute $\mathbb{Z}$-basis of such lattices. We exhibit the converse function $\pi_{\boldsymbol{\alpha}}^{-1}: \Gamma_{\boldsymbol{\alpha}} \longrightarrow \mathfrak{P}(\mathbf{v}, \mu, w)$ of the projection map $\pi_{\boldsymbol{\alpha}}$. This function admits a very simple expression when $\alpha_{3}=1$.

Several applications of generalized functionality are given in the rest of the paper, under the assumption that a functional vector exists with a coordinate equal to 1. We first apply in Section 5 the generalized functionality property to the enumeration of some local configurations, in particular we generalize results on $(m, n)$-cubes.

Section 6 provides one with two further applications of the generalized functionality property. We first extend Isabelle Debled-Rennesson's algorithm for computing the geometric representation of naive arithmetic discrete planes [Deb95] to any rational arithmetic discrete plane $\mathfrak{P}(\mathbf{v}, \mu, w)$ with $w-v_{3} \in$ $\operatorname{gcd}\left(v_{1}, v_{2}\right) \mathbb{Z}$. We then show that one can provide any arithmetic discrete plane $\mathfrak{P}(\mathbf{v}, \mu, w)$ with a structure of two-dimensional combinatorial manifold, under the assumption $w \in v_{1} \mathbb{Z}+v_{2} \mathbb{Z}+v_{3}$ and $w \geq \max \left\{\left|v_{1}\right|,\left|v_{2}\right|\right\}$.

Section 7 concludes the paper.

For clarity issues, we have chosen to work here in a three-dimensional space but all the results and methods presented extend in a natural way to $\mathbb{R}^{n}$, with $n \geq 2$, as well as to arithmetic discrete lines. Let us note that we have tried to make this paper essentially self-contained. This paper is an extended version of [BFJ05], but also contains some new applications of functionality.

\section{$2 \quad$ Arithmetic discrete planes}

In the present paper, the $\mathbb{R}$-vector space $\mathbb{R}^{3}$ is endowed with its canonical basis $\left\{\mathbf{e}_{1}, \mathbf{e}_{2}, \mathbf{e}_{3}\right\}$. Let $\mathbf{v}$ and $\mathbf{v}^{\prime}$ be two vectors of $\mathbb{R}^{3}$, their usual scalar product in $\mathbb{R}^{3}$ is denoted by $\left\langle\mathbf{v}, \mathbf{v}^{\prime}\right\rangle$. The subsets of all nonzero elements in $\mathbb{R}$ and $\mathbb{N}$ are denoted by $\mathbb{R}^{\star}$ and $\mathbb{N}^{\star}$ respectively.

Let $\mathbf{v} \in \mathbb{R}^{3}, \mu \in \mathbb{R}, w \in \mathbb{R}_{+}^{\star}$, and define:

$$
\mathfrak{P}(\mathbf{v}, \mu, w)=\left\{\mathbf{x} \in \mathbb{Z}^{3}, 0 \leq\langle\mathbf{v}, \mathbf{x}\rangle+\mu<w\right\}
$$


An arithmetic discrete plane is a subset $\mathcal{P}$ of $\mathbb{Z}^{3}$ such that:

$$
\exists(\mathbf{v}, \mu, w) \in \mathbb{R}^{3} \times \mathbb{R} \times \mathbb{R}_{+}^{*}, \quad \mathcal{P}=\mathfrak{P}(\mathbf{v}, \mu, w) .
$$

The following lemma provides us with basic properties of arithmetic discrete planes. In particular, the vector $\mathbf{v}$ in $(2)$ is shown to be unique up to scale. For $\mathbf{v}=\left(v_{1}, v_{2}, v_{3}\right) \in \mathbb{Z}^{3}$, let $\operatorname{gcd}\left\{v_{1}, v_{2}, v_{3}\right\}$ be denoted by $\operatorname{gcd}(\mathbf{v})$, and for $\mathbf{v} \in \mathbb{R}^{3}$, let the dimension of the $\mathbb{Q}$-vector space spanned by $\left\{v_{1}, v_{2}, v_{3}\right\}$ be denoted by $\operatorname{dim}_{\mathbb{Q}} \mathbf{v}$ (for instance, the following $\mathbf{v}$-values: $(1,2,3),(\sqrt{2}, 2 \sqrt{2}, 3 \sqrt{2})$, $(1, \sqrt{2}, 2 \sqrt{2})$, and $(1, \sqrt{2}, \sqrt{3})$ yield $\operatorname{dim}_{\mathbb{Q}} \mathbf{v}=1,1,2$, and 3 respectively).

Lemma 2 Let $\mathcal{P}=\mathfrak{P}(\mathbf{v}, \mu, w)$ be an arithmetic discrete plane with $(\mathbf{v}, \mu, w) \in$ $\mathbb{R}^{3} \times \mathbb{R} \times \mathbb{R}_{+}^{\star}$.

(i) If $\mathcal{P}=\mathfrak{P}\left(\mathbf{v}^{\prime}, \mu^{\prime}, w^{\prime}\right)$ then there exists $t \in \mathbb{R}$ such that $\mathbf{v}^{\prime}=t \mathbf{v}$.

(ii) The set $\{\langle\mathbf{v}, \mathbf{x}\rangle+\mu, \mathbf{x} \in \mathcal{P}\}$ is dense in $\left[0, w\left[\right.\right.$ if, and only if, $\operatorname{dim}_{\mathbb{Q}} \mathbf{v}>1$.

(iii) There exist $\mathbf{v}^{\prime} \in \mathbb{Z}^{3}$ with $\operatorname{gcd}\left(\mathbf{v}^{\prime}\right)=1, \mu^{\prime} \in \mathbb{Z}$, and $w^{\prime} \in \mathbb{N}^{\star}$ such that $\mathcal{P}=\mathfrak{P}\left(\mathbf{v}^{\prime}, \mu^{\prime}, w^{\prime}\right)$ if, and only if, $\operatorname{dim}_{\mathbb{Q}} \mathbf{v}=1$. If so, then $\operatorname{Card}\{\langle\mathbf{v}, \mathbf{x}\rangle+\mu, \mathbf{x} \in$ $\mathcal{P}\}=w^{\prime}$.

Proof. Let us assume $\operatorname{dim}_{\mathbb{Q}} \mathbf{v}>1$. There exist $i, j \in\{1,2,3\}$ such that $v_{i} \neq 0$ and $\frac{v_{j}}{v_{i}} \notin \mathbb{Q}$. By Kronecker's Approximation Theorem, the set $\{a t+b,(a, b) \in$ $\left.\mathbb{Z}^{2}\right\}$ is dense in $\mathbb{R}$ if $t \in \mathbb{R} \backslash \mathbb{Q}$, that is, for every interval $I \subset \mathbb{R}$, there exists $(a, b) \in \mathbb{Z}^{2}$ such that $a t+b \in I$. Let $I$ be an sub-interval of $[0, \omega[$; one has $\frac{v_{j}}{v_{i}} \notin \mathbb{Q}$; hence there exists $\left(x_{i}, x_{j}\right) \in \mathbb{Z}^{2}$ such that $x_{i}+x_{j} \frac{v_{j}}{v_{i}}+\frac{\mu}{v_{i}} \in \frac{I}{v_{i}}$, that is, $x_{i} v_{i}+x_{j} v_{j}+\mu \in I$. Hence the set $\{\langle\mathbf{v}, \mathbf{x}\rangle+\mu, \mathbf{x} \in \mathcal{P}\}$ is dense in $[0, w[$.

If $\operatorname{dim}_{\mathbb{Q}} \mathbf{v}=1$, then there exists $t \in \mathbb{R}_{+}$such that $t \mathbf{v} \in \mathbb{Z}^{3}$ and $\operatorname{gcd}(t \mathbf{v})=1$. A straightforward calculation shows that in (iii), one can take $\mathbf{v}^{\prime}=t \mathbf{v}, \mu^{\prime}=\lfloor t \mu\rfloor$, and $w^{\prime}=\lceil t w-t \mu\rceil+\lfloor t \mu\rfloor$. Furthermore note that the set $\left\{\mathbf{x} \in \mathbb{Z}^{3},\left\langle\mathbf{v}^{\prime}, \mathbf{x}\right\rangle+\mu^{\prime}=\right.$ $k\}$ is not empty if, and only if, $k \in \mathbb{Z}$. Thus $\operatorname{Card}\left\{\left\langle\mathbf{v}^{\prime}, \mathbf{x}\right\rangle+\mu^{\prime}, \mathbf{x} \in \mathcal{P}\right\}=w^{\prime}$, which completes the proof of (ii) and (iii) since the sets $\left\{\left\langle\mathbf{v}^{\prime}, \mathbf{x}\right\rangle+\mu^{\prime}, \mathbf{x} \in \mathcal{P}\right\}$ and $\{\langle\mathbf{v}, \mathbf{x}\rangle+\mu, \mathbf{x} \in \mathcal{P}\}$ are homothetical.

Let us next prove (i). First assume that $\mathcal{P}=\mathfrak{P}(\mathbf{v}, \mu, w)=\mathfrak{P}\left(\mathbf{v}^{\prime}, \mu^{\prime}, w^{\prime}\right)$ with the further condition $0 \leq \mu<w$, so that $\mathcal{P}$ contains $(0,0,0)$. Let $V$ be the $\mathbb{R}$-vector space spanned by $\left\{\mathbf{v}, \mathbf{v}^{\prime}\right\}$, and define:

$$
B=\left\{\mathbf{x} \in \mathbb{R}^{3}, 0 \leq\langle\mathbf{v}, \mathbf{x}\rangle+\mu<w\right\}, \quad B^{\prime}=\left\{\mathbf{x} \in \mathbb{R}^{3}, 0 \leq\left\langle\mathbf{v}^{\prime}, \mathbf{x}\right\rangle+\mu^{\prime}<w^{\prime}\right\} .
$$

Suppose $\operatorname{dim}_{\mathbb{R}} V=2$. Then $B \cap B^{\prime} \cap V$ is bounded (a parallelogram), so that its discrete subset $\mathcal{P} \cap V$ is finite. Let $\mathbf{x}=a \mathbf{v}+b \mathbf{v}^{\prime}$ with $(a, b) \in \mathbb{Z}^{2}$; then $\mathbf{x} \in \mathcal{P}$ if, and only if, $0 \leq\langle\mathbf{v}, \mathbf{v}\rangle a+\left\langle\mathbf{v}, \mathbf{v}^{\prime}\right\rangle b+\mu<w^{\prime}$. Therefore, $\mathcal{P} \cap V$ is the arithmetic discrete line with normal vector $\left(\langle\mathbf{v}, \mathbf{v}\rangle,\left\langle\mathbf{v}, \mathbf{v}^{\prime}\right\rangle\right)$, translation parameter $\mu$ and thickness $w^{\prime}$. Since it is not empty, it is not finite, a contradiction.

Finally, let us show that the conclusion still holds for arbitrary $\mu$. If $\operatorname{dim}_{\mathbb{Q}} \mathbf{v}=$ 1 , we may assume that $\mathbf{v} \in \mathbb{Z}^{3}$ with $\operatorname{gcd}(\mathbf{v})=1$ and $\mu \in \mathbb{Z}$. By using Bezout's Lemma let us choose $\mathbf{u}$ in $\mathbb{Z}^{3}$ such that $\langle\mathbf{u}, \mathbf{v}\rangle=1$ and let $\mathbf{u}^{\prime}=\mu \mathbf{u}$. If $\operatorname{dim}_{\mathbb{Q}} \mathbf{v}>$ 1 , choose $\mathbf{u}^{\prime} \in \mathbb{Z}^{3}$ such that $\left\langle\mathbf{u}^{\prime}, \mathbf{v}\right\rangle \in\left[\mu, \mu+w^{\prime}\left[\right.\right.$. Let $\tau: \mathbb{R}^{3} \rightarrow \mathbb{R}^{3}$ be the trans- 
lation by $-\mathbf{u}^{\prime}$, we have $\tau(\mathcal{P})=\mathfrak{P}\left(\mathbf{v}, \mu-\left\langle\mathbf{u}^{\prime}, \mathbf{v}\right\rangle, w\right)=\mathfrak{P}\left(\mathbf{v}^{\prime}, \mu-\left\langle\mathbf{u}^{\prime}, \mathbf{v}\right\rangle, w^{\prime}\right)$. Since $0 \leq \mu-\left\langle\mathbf{u}^{\prime}, \mathbf{v}\right\rangle<w^{\prime}$, we still conclude that $\mathbf{v}$ and $\mathbf{v}^{\prime}$ are colinear.

An arithmetic discrete plane that may be represented by $\mathcal{P}=\mathfrak{P}(\mathbf{v}, \mu, w)$ with $\operatorname{dim}_{\mathbb{Q}} \mathbf{V}=1$ is called rational, otherwise it is called irrational. According to Lemma 2, the distinction is exclusive. From now on, we shall agree that any representation $\mathfrak{P}(\mathbf{v}, \mu, w)$ of a rational arithmetic discrete plane satisfies:

$$
\mathbf{v} \in \mathbb{Z}^{3} \text { and } \operatorname{gcd}(\mathbf{v})=1, \quad \mu \in \mathbb{Z}, \quad w \in \mathbb{N}^{\star} .
$$

Exactly two such representations exist for a rational arithmetic discrete plane:

$$
\mathfrak{P}(\mathbf{v}, \mu, w)=\mathfrak{P}(-\mathbf{v}, w-1-\mu, w)
$$

Indeed, only two choices are allowed for $\mathbf{v}$ by Lemma 2 (i), and $w$ is unique by Lemma 2 (iii). Then other $\mu$-values are easily ruled out. For an irrational arithmetic discrete plane, constraint $\langle\mathbf{v}, \mathbf{v}\rangle=1$ and Lemma 2 (ii) result in similar conclusions: There are at most two representations of an irrational arithmetic discrete plane $\mathfrak{P}(\mathbf{v}, \mu, w)$, the second one being $\mathfrak{P}(-\mathbf{v}, w-\mu, w)$ and happening if, and only if, neither $\mu$ nor $w-\mu$ belong to the $\mathbb{Q}$-vector space spanned by $\left\{v_{1}, v_{2}, v_{3}\right\}$.

Some among rational arithmetic discrete planes are usually given particular names [Rev91,DR94,Deb95,Fra96]. Let $\mathbf{v}=\left(v_{1}, v_{2}, v_{3}\right) \in \mathbb{Z}^{3}$; if $w=$ $\max \left\{\left|v_{1}\right|,\left|v_{2}\right|,\left|v_{3}\right|\right\}$ (resp. $\left.w=\left|v_{1}\right|+\left|v_{2}\right|+\left|v_{3}\right|\right)$, then $\mathcal{P}=\mathfrak{P}(\mathbf{v}, \mu, w)$ is called naive (resp. standard). It is important to remark that, according to the above discussion, these definitions do not depend on any specific representation of $\mathcal{P}$.

Let us next recall a classical property of naive discrete planes with normal vector $\mathbf{v}$. For a given $\boldsymbol{\alpha} \in \mathbb{Z}^{3}$, let $\pi_{\boldsymbol{\alpha}}^{\perp}: \mathbb{R}^{3} \rightarrow\left\{\mathbf{x} \in \mathbb{R}^{3}:\langle\boldsymbol{\alpha}, \mathbf{x}\rangle=0\right\}$ stand for the orthogonal projection map onto the plane $\langle\boldsymbol{\alpha}, \mathbf{x}\rangle=0$. We still denote by $\pi_{\alpha}^{\perp}$ its restriction to any subset of $\mathbb{R}^{3}$ or its corestriction to any subset of the plane $\langle\boldsymbol{\alpha}, \mathbf{x}\rangle=0$, as for instance $\pi_{\boldsymbol{\alpha}}^{\perp}: \mathfrak{P}(\mathbf{v}, \mu, w) \rightarrow \pi_{\boldsymbol{\alpha}}^{\perp}\left(\mathbb{Z}^{3}\right)$.

Theorem 3 ([Rev91]) Let $\mathcal{P}=\mathfrak{P}(\mathbf{v}, \mu, w)$ be a naive arithmetic discrete plane, with $\mathbf{v}=\left(v_{1}, v_{2}, v_{3}\right) \in \mathbb{R}^{3}$. If $\left|v_{i}\right|=w$, for $i=1,2$ or 3 , then $\mathcal{P}$ is in bijection with the integer points of the plane $\left\langle\mathbf{e}_{\mathbf{i}}, \mathbf{x}\right\rangle=0$ by the projection map $\pi_{\mathbf{e}_{\mathbf{i}}}^{\perp}$.

The plane $\left\langle\mathbf{e}_{\mathbf{i}}, \mathbf{x}\right\rangle=0$ in Theorem 3 is called a functional plane of $\mathcal{P}$. An analogous result holds for standard discrete planes:

Theorem 4 ([Fra96,BV00]) Let $\mathcal{P}$ be a standard discrete plane and let $\boldsymbol{\alpha}=$ $\mathbf{e}_{\mathbf{1}}+\mathbf{e}_{\mathbf{2}}+\mathbf{e}_{\mathbf{3}}$. Then the map $\pi_{\boldsymbol{\alpha}}^{\perp}: \mathcal{P} \rightarrow \pi_{\boldsymbol{\alpha}}^{\perp}\left(\mathbb{Z}^{3}\right)$ is a bijection.

Both results are extended in several directions in the following section. 


\section{Generalized functionality}

In both cases investigated in Theorems 3 and 4 the following property holds: there exists a vector $\boldsymbol{\alpha} \in \mathbb{Z}^{3}$ such that the projection map $\pi_{\boldsymbol{\alpha}}^{\perp}: \mathfrak{P}(\mathbf{v}, \mu, w) \rightarrow$ $\pi_{\boldsymbol{\alpha}}^{\perp}\left(\mathbb{Z}^{3}\right)$ is a bijection, and $\langle\boldsymbol{\alpha}, \mathbf{v}\rangle=w$. In this section, it is extended to a large class of arithmetic discrete planes by means of functional directions:

Definition $5 A$ vector $\boldsymbol{\alpha} \in \mathbb{Z}^{3}$ is called functional for an arithmetic discrete plane $\mathfrak{P}(\mathbf{v}, \mu, w)$ if it satisfies conditions $\operatorname{gcd}(\boldsymbol{\alpha})=1$ and $\langle\boldsymbol{\alpha}, \mathbf{v}\rangle=w$.

According to Lemma 2, functionality of a vector $\boldsymbol{\alpha} \in \mathbb{Z}^{3}$ for an arithmetic discrete plane $\mathfrak{P}(\mathbf{v}, \mu, w)$ does not depend on the representation of the latter.

Furthermore, we improve Theorems 3 and 4 by showing that the vectors $\boldsymbol{\alpha} \in$ $\mathbb{Z}^{3}$ providing us with bijective projections $\pi_{\alpha}^{\perp}$ are exactly the functional ones (to be precise, this statement also holds for $-\alpha$ if $\alpha$ is functional since $\operatorname{gcd}(\boldsymbol{\alpha})=$ $\operatorname{gcd}(-\boldsymbol{\alpha})$ and $\left.\pi_{\boldsymbol{\alpha}}^{\perp}=\pi_{-\boldsymbol{\alpha}}^{\perp}\right)$.

Theorem 6 Let $\mathcal{P}=\mathfrak{P}(\mathbf{v}, \mu, w)$ be an arithmetic discrete plane, and let $\boldsymbol{\alpha} \in$ $\mathbb{Z}^{3}$ be such that $\operatorname{gcd}(\boldsymbol{\alpha})=1$. Then the map $\pi_{\boldsymbol{\alpha}}^{\perp}: \mathcal{P} \rightarrow \pi_{\boldsymbol{\alpha}}^{\perp}\left(\mathbb{Z}^{3}\right)$ is a bijection if, and only if, $|\langle\boldsymbol{\alpha}, \mathbf{v}\rangle|=w$.

Proof. Throughout the proof, we assume w.l.o.g. that $\langle\boldsymbol{\alpha}, \mathbf{v}\rangle \geq 0$. Let $\mathbf{x}, \mathbf{x}^{\prime} \in$ $\mathbb{Z}^{3}$, then $\pi_{\boldsymbol{\alpha}}^{\perp}(\mathbf{x})=\pi_{\boldsymbol{\alpha}}^{\perp}\left(\mathbf{x}^{\prime}\right)$ if, and only if, there exists $\left(k, k^{\prime}\right) \in \mathbb{Z}^{2}$ such that $k^{\prime}\left(\mathbf{x}^{\prime}-\mathbf{x}\right)=k \boldsymbol{\alpha}$. Since $\operatorname{gcd}(\boldsymbol{\alpha})=1, k^{\prime}$ divides $k$. In other words, $\pi_{\boldsymbol{\alpha}}^{\perp}(\mathbf{x})=$ $\pi_{\boldsymbol{\alpha}}^{\perp}\left(\mathbf{x}^{\prime}\right)$ if, and only if, there exists $c \in \mathbb{Z}$ such that $\mathbf{x}^{\prime}=\mathbf{x}+c \boldsymbol{\alpha}$. Moreover, $\mathbf{x}+$ $c \boldsymbol{\alpha} \in \mathcal{P}$ if, and only if, $\frac{-(\langle\mathbf{x}, \mathbf{v}\rangle+\mu)}{\langle\boldsymbol{\alpha}, \mathbf{v}\rangle} \leq c<\frac{w-(\langle\mathbf{x}, \mathbf{v}\rangle+\mu)}{\langle\boldsymbol{\alpha}, \mathbf{v}\rangle}$. Accordingly, if $|\langle\boldsymbol{\alpha}, \mathbf{v}\rangle|=w$ then $c$ is allowed to take exactly one value, so that $\pi_{\boldsymbol{\alpha}}^{\perp}: \mathcal{P} \rightarrow \pi_{\boldsymbol{\alpha}}^{\perp}\left(\mathbb{Z}^{3}\right)$ is a bijection.

Conversely assume that $\pi_{\boldsymbol{\alpha}}^{\perp}: \mathcal{P} \rightarrow \pi_{\boldsymbol{\alpha}}^{\perp}\left(\mathbb{Z}^{3}\right)$ is a bijection. If $\mathcal{P}$ is rational, take $\mathbf{x} \in \mathbb{Z}^{3}$ such that $\langle\mathbf{x}, \mathbf{v}\rangle+\mu=0$ by using Bezout's Lemma. On the one hand, $\mathbf{x} \in \mathcal{P}$ and $\langle\mathbf{x}+\boldsymbol{\alpha}, \mathbf{v}\rangle+\mu=\langle\boldsymbol{\alpha}, \mathbf{v}\rangle \geq 0$. Moreover, $\pi_{\boldsymbol{\alpha}}^{\perp}(\mathbf{x}+\boldsymbol{\alpha})=\pi_{\boldsymbol{\alpha}}^{\perp}(\mathbf{x})$. Since $\pi_{\boldsymbol{\alpha}}^{\perp}$ is one-to-one, $\mathbf{x}+\boldsymbol{\alpha} \notin \mathcal{P}$, thus $\langle\boldsymbol{\alpha}, \mathbf{v}\rangle \geq w$. On the other hand, suppose that $\langle\boldsymbol{\alpha}, \mathbf{v}\rangle>w$ and choose $\mathbf{y} \in \mathbb{Z}^{3}$ such that $\langle\mathbf{y}, \mathbf{v}\rangle+\mu=-1$. Then, for $c \in \mathbb{Z},\langle\mathbf{y}+c \boldsymbol{\alpha}, \mathbf{v}\rangle+\mu=c\langle\boldsymbol{\alpha}, \mathbf{v}\rangle-1$, which is negative if $c \leq 0$, and larger than or equal to $w-1$ if $c>0$. Since $\pi_{\alpha}^{\perp}$ is onto, this cannot happen. Thus $\langle\boldsymbol{\alpha}, \mathbf{v}\rangle=w$.

If $\mathcal{P}$ is irrational, we parallel the previous proof by using a density argument instead of Bezout's Lemma. Consider any $\varepsilon \in \mathbb{R}$ and choose $\mathbf{x} \in \mathbb{Z}^{3}$ such that $\langle\mathbf{x}, \mathbf{v}\rangle+\mu \in[0, \varepsilon[$. We obtain as above $\langle\boldsymbol{\alpha}, \mathbf{v}\rangle>w-\varepsilon$, thus $\langle\boldsymbol{\alpha}, \mathbf{v}\rangle \geq$ $w$. Suppose $\langle\boldsymbol{\alpha}, \mathbf{v}\rangle>w$, let $\nu=\langle\boldsymbol{\alpha}, \mathbf{v}\rangle-w$, and choose $\mathbf{y} \in \mathbb{Z}^{3}$ such that $\langle\mathbf{y}, \mathbf{v}\rangle+\mu \in]-\nu / 2,0[$. Then, for $c \in \mathbb{Z},\langle\mathbf{y}+c \boldsymbol{\alpha}, \mathbf{v}\rangle+\mu=c\langle\boldsymbol{\alpha}, \mathbf{v}\rangle+\langle\mathbf{y}, \mathbf{v}\rangle+\mu$. 
This quantity is negative if $c \leq 0$, and is larger $w$, if $c>0$.

If $\boldsymbol{\alpha}$ is a functional vector for an arithmetic discrete plane $\mathcal{P}$, then Theorem 6 states that, looking at $\mathcal{P}$ along this direction, one can see all its points as if they were on the plane $\left\{\mathbf{x} \in \mathbb{R}^{3},\langle\boldsymbol{\alpha}, \mathbf{x}\rangle=0\right\}$. We show later that a natural regular lattice structure emerges from this point of view. Let us first complete Theorem 6 by relaxing condition $\operatorname{gcd}(\boldsymbol{\alpha})=1$ for rational planes.

Corollary 7 Let $\mathcal{P}=\mathfrak{P}(\mathbf{v}, \mu, w)$ be a rational arithmetic discrete plane and $\langle\boldsymbol{\alpha}, \mathbf{v}\rangle=w$. If $\operatorname{gcd}(\boldsymbol{\alpha})=d$, where $d \in \mathbb{N}$ divides $w$, then $\pi_{\boldsymbol{\alpha}}^{\perp}: \mathcal{P} \rightarrow \pi_{\boldsymbol{\alpha}}^{\perp}\left(\mathbb{Z}^{3}\right)$ satisfies, for each $\mathbf{p} \in \pi_{\boldsymbol{\alpha}}^{\perp}\left(\mathbb{Z}^{3}\right)$,

$$
\left(\pi_{\alpha}^{\perp}\right)^{-1}(\{\mathbf{p}\})=\left\{\mathbf{x}_{\mathbf{p}}+k \frac{\boldsymbol{\alpha}}{d}, 0 \leq k \leq d-1\right\}
$$

where $\mathbf{x}_{\mathbf{p}}$ is the unique solution in $\mathfrak{P}(\mathbf{v}, \mu, w / d)$ of $\pi_{\boldsymbol{\alpha}}^{\perp}(\mathbf{x})=\mathbf{p}$.

Proof. Let $\mathcal{P}_{k}=\mathfrak{P}(\mathbf{v}, \mu-k w / d, w / d)$ and let us write $\mathcal{P}$ as the disjoint union $\mathcal{P}=\bigcup_{k=0}^{d-1} \mathcal{P}_{k}$. One has $\pi_{\boldsymbol{\alpha} / d}^{\perp}=\pi_{\boldsymbol{\alpha}}^{\perp}$. By Theorem 6, the projection $\pi_{\boldsymbol{\alpha}}^{\perp}: \mathcal{P}_{k} \rightarrow$ $\pi_{\boldsymbol{\alpha}}^{\perp}\left(\mathbb{Z}^{3}\right)$ is one-to-one for each $k$ between 0 and $d-1$, so that the cardinality of $\left(\pi_{\boldsymbol{\alpha}}^{\perp}\right)^{-1}(\{\mathbf{p}\})$ does not exceed $d$. Moreover, given $\mathbf{p} \in \pi_{\boldsymbol{\alpha}}^{\perp}\left(\mathbb{Z}^{3}\right), \mathbf{x}_{\mathbf{p}}$ is welldefined. Since $\langle\boldsymbol{\alpha} / d, \mathbf{v}\rangle=w / d$, for $0 \leq k \leq d-1$, each point $\mathbf{x}_{\mathbf{p}}+k \frac{\boldsymbol{\alpha}}{d}$ is in $\mathcal{P}_{k}$. Whence the result.

In particular, taking $d=w$ in Corollary 7 one obtains:

Corollary 8 Let $\mathcal{P}=\mathfrak{P}(\mathbf{v}, \mu, w)$ be a rational arithmetic discrete plane and $\langle\boldsymbol{\alpha}, \mathbf{v}\rangle=w$. If $\operatorname{gcd}(\boldsymbol{\alpha})=w$, then $\mathcal{P}$ is the disjoint union $\mathcal{P}=\bigcup_{k=0}^{w-1}\left(\mathcal{P}_{0}+k \frac{\boldsymbol{\alpha}}{w}\right)$, where $\mathcal{P}_{0}$ is the discrete plane $\left\{\mathbf{x} \in \mathbb{Z}^{3},\langle v, x\rangle+\mu=0\right\}$.

In case of an irrational discrete plane $\mathfrak{P}(\mathbf{v}, \mu, w)$, there is no reason for a vector $\boldsymbol{\alpha} \in \mathbb{Z}^{3}$ to exist satisfying $\langle\boldsymbol{\alpha}, \mathbf{v}\rangle=w-$ consider $\left\{v_{1}, v_{2}, v_{3}, w\right\}$ spanning a 4-dimensional subspace of the $\mathbb{Q}$-vector space $\mathbb{R}$. Nevertheless, if $\mathfrak{P}(\mathbf{v}, \mu, w)$ is rational then we deduce from Bezout's Lemma that such an $\boldsymbol{\alpha}$ exists. We next prove that it can be chosen such that $\operatorname{gcd}(\boldsymbol{\alpha})=1$.

Proposition 9 Any rational arithmetic discrete plane $\mathfrak{P}(\mathbf{v}, \mu, w)$ has a functional vector.

Proof. Let $\boldsymbol{\beta}, \mathbf{u} \in \mathbb{Z}^{3}$ such that $\langle\boldsymbol{\beta}, \mathbf{v}\rangle=1$ and $\langle\mathbf{u}, \mathbf{v}\rangle=0$. Let $d=\operatorname{gcd}(\mathbf{u})$ and let $\boldsymbol{\alpha}=w \boldsymbol{\beta}+\mathbf{u} / d$. An easy computation gives $\langle\boldsymbol{\alpha}, \mathbf{v}\rangle=w$ and $\operatorname{gcd}(\boldsymbol{\alpha})=1$.

Proposition 9 is illustrated in Figure 1, in the case of a discrete line for a better visualisation. Moreover, rather than projecting on the line $\left\{\mathbf{x} \in \mathbb{R}^{2},\langle\boldsymbol{\alpha}, \mathbf{x}\rangle=\right.$ $0\}$ we prefer projecting on the line $\left\{\mathbf{x} \in \mathbb{R}^{2},\left\langle\mathbf{e}_{\mathbf{2}}, \mathbf{x}\right\rangle=0\right\}$, which amounts to 
the same as far as bijectivity is concerned.

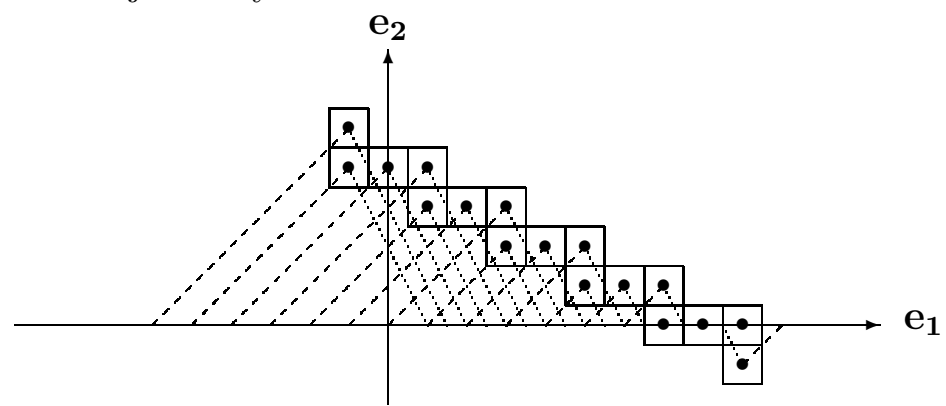

Fig. 1. Generalized functionality: the discrete line $0 \leq x_{1}+2 x_{2}-7<3$ is projected on $\left\{\mathbf{x} \in \mathbb{R}^{2},\left\langle\mathbf{e}_{2}, \mathbf{x}\right\rangle=0\right\}$ along $\boldsymbol{\alpha}=\mathbf{e}_{1}+\mathbf{e}_{2}$ and $\boldsymbol{\alpha}=-\mathbf{e}_{\mathbf{1}}+2 \mathbf{e}_{\mathbf{2}}$.

With Figure 1 again, note that the projection is not one-to-one for $\boldsymbol{\alpha}=\mathbf{e}_{\mathbf{2}}$ since $\left\langle\mathbf{e}_{\mathbf{2}}, \mathbf{v}\right\rangle=2<w$, and it is not onto for $\boldsymbol{\alpha}=2 \mathbf{e}_{\mathbf{1}}+\mathbf{e}_{\mathbf{1}}$ since $\langle\boldsymbol{\alpha}, \mathbf{v}\rangle=4>w$ (see the proof of Th. 6).

\section{Functional lattices}

Let us next study how arithmetic discrete planes can be recoded in a functional way by a regular two-dimensional lattice, despite their three-dimensional structure. Let $\mathcal{P}=\mathfrak{P}(\mathbf{v}, \mu, w)$ be an arithmetic discrete plane and let $\boldsymbol{\alpha} \in \mathbb{Z}^{3}$ be functional for $\mathcal{P}$, that is, $\operatorname{gcd}\{\boldsymbol{\alpha}\}=1$ and $\langle\boldsymbol{\alpha}, \mathbf{v}\rangle=w$ (in case $\mathcal{P}$ is rational, the existence of such a vector $\boldsymbol{\alpha}$ is ensured by Proposition 9). Since one of the coordinates $\alpha_{i}$, for $i \in\{1,2,3\}$, is non-zero, then we assume $\alpha_{3} \neq 0$ without loss of generality.

Accordingly, we shall from now on prefer to $\pi_{\boldsymbol{\alpha}}^{\perp}$ the projection $\pi_{\boldsymbol{\alpha}}$ along $\boldsymbol{\alpha}$ onto the plane $\left\{\mathbf{x} \in \mathbb{R}^{3},\left\langle\mathbf{e}_{\mathbf{3}}, \mathbf{x}\right\rangle=0\right\}$, as it was done in Figure 1. Indeed Theorem 6 also holds for $\pi_{\boldsymbol{\alpha}}$ since $\pi_{\boldsymbol{\alpha}}$ is a bijection if, and only if, $\pi_{\boldsymbol{\alpha}}^{\perp}$ is also a bijection.

First note that, since $\pi_{\boldsymbol{\alpha}}(\boldsymbol{\alpha})=0$ and $\pi_{\boldsymbol{\alpha}}\left(\mathbf{e}_{\mathbf{i}}\right)=\mathbf{e}_{\mathbf{i}}$ for $i \in\{1,2\}$, one has $\alpha_{3} \pi_{\boldsymbol{\alpha}}\left(\mathbf{e}_{\mathbf{3}}\right)=-\alpha_{1} \mathbf{e}_{\mathbf{1}}-\alpha_{2} \mathbf{e}_{\mathbf{2}}$. Then, for all $\mathbf{x}=\left(x_{1}, x_{2}, x_{3}\right) \in \mathbb{Z}^{3}$, a straightforward calculation gives:

$$
\pi_{\boldsymbol{\alpha}}(\mathbf{x})=\frac{\alpha_{3} x_{1}-\alpha_{1} x_{3}}{\operatorname{gcd}\left\{\alpha_{1}, \alpha_{3}\right\}} \mathbf{f}_{\mathbf{1}}+\frac{\alpha_{3} x_{2}-\alpha_{2} x_{3}}{\operatorname{gcd}\left\{\alpha_{2}, \alpha_{3}\right\}} \mathbf{f}_{\mathbf{2}}, \quad \text { where } \mathbf{f}_{\mathbf{i}}=\frac{\operatorname{gcd}\left\{\alpha_{i}, \alpha_{3}\right\}}{\alpha_{3}} \mathbf{e}_{\mathbf{i}}
$$

We deduce from (6) that $\Gamma_{\boldsymbol{\alpha}}=\pi_{\boldsymbol{\alpha}}\left(\mathbb{Z}^{3}\right)=\pi_{\boldsymbol{\alpha}}(\mathcal{P})$ is a subset of $\mathbb{Z} \mathbf{f}_{\mathbf{1}}+\mathbb{Z} \mathbf{f}_{\mathbf{2}}$. The description of $\Gamma_{\boldsymbol{\alpha}}$ is made more precise below. In particular, it is shown to be a lattice, which shall be called a functional lattice of $\mathcal{P}$. This definition generalizes the concept of functionality defined for naive arithmetic discrete planes as a projection onto the integer points of one of the coordinate planes.

Let us first give a characterization of the points in $\Gamma_{\boldsymbol{\alpha}}$. 
Theorem 10 Assume that $\operatorname{gcd}\{\boldsymbol{\alpha}\}=1, \alpha_{3} \neq 0$, and, for $i \in\{1,2,3\}$, let

$$
\delta_{i}=\frac{\alpha_{i}}{\prod_{j \neq i} \operatorname{gcd}\left\{\alpha_{i}, \alpha_{j}\right\}} \in \mathbb{Z}
$$

A point with coordinates $\left(y_{1}, y_{2}\right) \in \mathbb{Z}^{2}$ in the basis $\left\{\mathbf{f}_{\mathbf{1}}, \mathbf{f}_{\mathbf{2}}\right\}$ belongs to $\Gamma_{\boldsymbol{\alpha}}$ if, and only if, $\delta_{3}$ divides $\delta_{2} y_{1}-\delta_{1} y_{2}$.

Proof. According to (6), we have to show that the Diophantine system

$$
\left\{\begin{array}{l}
\operatorname{gcd}\left\{\alpha_{1}, \alpha_{3}\right\} y_{1}=\alpha_{3} x_{1}-\alpha_{1} x_{3} \\
\operatorname{gcd}\left\{\alpha_{2}, \alpha_{3}\right\} y_{2}=\alpha_{3} x_{2}-\alpha_{2} x_{3}
\end{array}\right.
$$

has a solution $\left(x_{1}, x_{2}, x_{3}\right) \in \mathbb{Z}^{3}$ if, and only if, $\delta_{2} y_{1} \equiv \delta_{1} y_{2} \bmod \delta_{3}$. The condition is clearly necessary: just rewrite (7) as

$$
\left\{\begin{array}{l}
y_{1}=\delta_{3} \operatorname{gcd}\left\{\alpha_{2}, \alpha_{3}\right\} x_{1}-\delta_{1} \operatorname{gcd}\left\{\alpha_{1}, \alpha_{2}\right\} x_{3} \\
y_{2}=\delta_{3} \operatorname{gcd}\left\{\alpha_{1}, \alpha_{3}\right\} x_{2}-\delta_{2} \operatorname{gcd}\left\{\alpha_{1}, \alpha_{2}\right\} x_{3}
\end{array}\right.
$$

Conversely, let us first note that each of the conditions $\alpha_{1}=0, \alpha_{2}=0$, or $\alpha_{3}=1$ easily yields solutions to (7). Assume none of them holds and denote, for $i=1,2$, by $\gamma_{i}$ the inverse of $\delta_{i} \operatorname{gcd}\left\{\alpha_{1}, \alpha_{2}\right\}$ modulo $\alpha_{3} / \operatorname{gcd}\left\{\alpha_{i}, \alpha_{3}\right\}$. Note that $\gamma_{i} \delta_{i} \operatorname{gcd}\left\{\alpha_{1}, \alpha_{2}\right\}$ is also 1 modulo $\delta_{3}$. Therefore, $\delta_{2} y_{1} \equiv \delta_{1} y_{2} \bmod \delta_{3}$ implies that $\gamma_{1} y_{1} \equiv \gamma_{2} y_{2} \bmod \delta_{3}$. Now let $\ell \in \mathbb{Z}$ such that $\gamma_{1} y_{1}-\gamma_{2} y_{2}=\ell \delta_{3}$, choose $p, q \in \mathbb{Z}$ such that $\operatorname{gcd}\left\{\alpha_{2}, \alpha_{3}\right\} p-\operatorname{gcd}\left\{\alpha_{1}, \alpha_{3}\right\} q=\ell$, and set:

$$
x_{3}=-\gamma_{1} y_{1}+p \delta_{3} \operatorname{gcd}\left\{\alpha_{2}, \alpha_{3}\right\}=-\gamma_{2} y_{2}+q \delta_{3} \operatorname{gcd}\left\{\alpha_{1}, \alpha_{3}\right\} \text {. }
$$

For $i=1,2$, we have $\delta_{i} \operatorname{gcd}\left\{\alpha_{1}, \alpha_{2}\right\} x_{3}+y_{i} \equiv 0 \bmod \alpha_{3} / \operatorname{gcd}\left\{\alpha_{i}, \alpha_{3}\right\}$. Setting

$$
x_{1}=\frac{\delta_{1} \operatorname{gcd}\left\{\alpha_{1}, \alpha_{2}\right\} x_{3}+y_{1}}{\delta_{3} \operatorname{gcd}\left\{\alpha_{2}, \alpha_{3}\right\}}, \quad x_{2}=\frac{\delta_{2} \operatorname{gcd}\left\{\alpha_{1}, \alpha_{2}\right\} x_{3}+y_{2}}{\delta_{3} \operatorname{gcd}\left\{\alpha_{1}, \alpha_{3}\right\}},
$$

we thus get a solution $\left(x_{1}, x_{2}, x_{3}\right) \in \mathbb{Z}^{3}$ of $(7)$.

Corollary 11 We have $\Gamma_{\boldsymbol{\alpha}}=\mathbb{Z} \mathbf{g}_{1}+\mathbb{Z} \mathbf{g}_{\mathbf{2}}$, with

$$
\mathbf{g}_{1}=\delta_{1} \mathbf{f}_{1}+\delta_{2} \mathbf{f}_{2}, \quad \mathbf{g}_{2}=u \mathbf{f}_{1}+v \mathbf{f}_{2},
$$

where $(u, v) \in \mathbb{Z}^{2}$ satisfies $\delta_{1} v-\delta_{2} u=\delta_{3}$.

Proof. Theorem 10 states that $\Gamma_{\boldsymbol{\alpha}}$ is the union, for $\ell$ running through $\mathbb{Z}$, of the subsets $\Lambda_{\ell}=\left\{y_{1} \mathbf{f}_{1}+y_{2} \mathbf{f}_{\mathbf{2}},\left(y_{1}, y_{2}\right) \in \mathbb{Z}^{2}, \delta_{1} y_{2}-\delta_{2} y_{1}=\ell \delta_{3}\right\}$ of the plane $\left\{\mathbf{x} \in \mathbb{R}^{3},\left\langle\mathbf{e}_{\mathbf{3}}, \mathbf{x}\right\rangle=0\right\}$. Since $\operatorname{gcd}\left\{\delta_{1}, \delta_{2}\right\}=1$ by definition, no set $\Lambda_{\ell}$ is empty. 
It follows that $\Gamma_{\boldsymbol{\alpha}}$ is a sublattice of $\mathbb{Z}^{2}$, a basis of which may consist in a basis of $\Lambda_{0}$ together with any vector shifting $\Lambda_{0}$ to $\Lambda_{1}$.

Another basis for $\Gamma_{\boldsymbol{\alpha}}$ is of interest because direction $e_{2}$ is conserved, namely:

$$
\mathbf{h}_{1}=\mathbf{f}_{\mathbf{1}}+s \delta_{2} \operatorname{gcd}\left\{\alpha_{1}, \alpha_{2}\right\} \mathbf{f}_{\mathbf{2}}, \quad \mathbf{h}_{\mathbf{2}}=\delta_{3} \mathbf{f}_{\mathbf{2}}
$$

where $s$ is any integer satisfying $s \alpha_{1}+t \alpha_{3}=\operatorname{gcd}\left\{\alpha_{1}, \alpha_{3}\right\}$, together with a fitting integer $t$. We leave it to the reader to check that:

$$
\left(\begin{array}{l}
\mathbf{g}_{\mathbf{1}} \\
\mathbf{g}_{\mathbf{2}}
\end{array}\right)=A\left(\begin{array}{l}
\mathbf{h}_{\mathbf{1}} \\
\mathbf{h}_{\mathbf{2}}
\end{array}\right), A=\left(\begin{array}{cc}
\delta_{1} & t \delta_{2} \operatorname{gcd}\left\{\alpha_{2}, \alpha_{3}\right\} \\
u & s \operatorname{gcd}\left\{\alpha_{1}, \alpha_{2}\right\}+v t \operatorname{gcd}\left\{\alpha_{2}, \alpha_{3}\right\}
\end{array}\right), \operatorname{det} A=1 .
$$

Considering an arithmetic discrete plane $\mathcal{P}$ with functional lattice $\Gamma_{\boldsymbol{\alpha}}$, a natural question arises: Given an element $\mathbf{y} \in \Gamma_{\boldsymbol{\alpha}}$, how to recover the unique vector $\mathbf{x} \in \mathcal{P}$ such that $\pi_{\boldsymbol{\alpha}}(\mathbf{x})=\mathbf{y} ?$

The height of $\mathcal{P}$ at $\mathbf{y} \in \Gamma_{\boldsymbol{\alpha}}$ is defined as the third coordinate $x_{3}$ of $\mathbf{x}=\pi_{\boldsymbol{\alpha}}^{-1}(\mathbf{y})$ and it is denoted by $H_{\mathcal{P}, \boldsymbol{\alpha}}(\mathbf{y})$. Thus $H_{\mathcal{P}, \boldsymbol{\alpha}}$ maps $\Gamma_{\boldsymbol{\alpha}}$ to $\mathbb{Z}$. According to (9), the height of $\mathcal{P}$ at $\mathbf{y}$ entirely determines $\pi_{\boldsymbol{\alpha}}^{-1}(\mathbf{y})$. It can be computed as follows.

Theorem 12 Let $\Gamma_{\boldsymbol{\alpha}}$ be a functional lattice of $\mathcal{P}=\mathfrak{P}(\mathbf{v}, \mu, w)$ and let $\mathbf{y} \in \Gamma_{\boldsymbol{\alpha}}$ with $\mathbf{y}=y_{1} \mathbf{f}_{1}+y_{2} \mathbf{f}_{\mathbf{2}}$. Then

$$
H_{\mathcal{P}, \alpha}(\mathbf{y})=-\lfloor m / w\rfloor+r
$$

where

$$
m=v_{1} y_{1} \operatorname{gcd}\left\{\alpha_{1}, \alpha_{3}\right\}+v_{2} y_{2} \operatorname{gcd}\left\{\alpha_{2}, \alpha_{3}\right\}+\mu \alpha_{3},
$$

and $r \in\left\{0, \ldots, \alpha_{3}-1\right\}$ is given by any of the following expressions:

(i) $r \equiv\lfloor-m / w\rfloor-a y_{1} \operatorname{gcd}\left\{\alpha_{1}, \alpha_{3}\right\}-b y_{2} \operatorname{gcd}\left\{\alpha_{2}, \alpha_{3}\right\} \bmod \alpha_{3}$, where $a, b$ are any integers such that $a \alpha_{1}+b \alpha_{2} \equiv 1 \bmod \alpha_{3}$.

(ii) If $\operatorname{gcd}\left\{w, \alpha_{3}\right\}=1$ then $r \equiv\lfloor m / w\rfloor-m / w \bmod \alpha_{3}$.

(iii) If $\alpha_{3}=1$ then $r=0$.

Proof. Let $\mathbf{x}=\left(x_{1}, x_{2}, x_{3}\right) \in \mathcal{P}$ and let $\mathbf{y}=\pi_{\boldsymbol{\alpha}}(\mathbf{x})$. According to (7) and $\langle\boldsymbol{\alpha}, \mathbf{v}\rangle=w$, we have

$$
\alpha_{3}\langle\mathbf{v}, \mathbf{x}\rangle=m+x_{3} w
$$

where $m$ is given by (14). Substituting in (1) and dividing each member by $w$ result in

$$
0 \leq \frac{m}{w}+x_{3}<\alpha_{3}
$$

Therefore, there exists a unique integer $r$ such that $0 \leq r \leq \alpha_{3}-1$ and $x_{3}=-\lfloor m / w\rfloor+r$. In particular, (iii) holds. Since $\operatorname{gcd}(\boldsymbol{\alpha})=1$, integers $a$ and 
$b$ can be found such that $a \alpha_{1}+b \alpha_{2} \equiv 1 \bmod \alpha_{3}$. A linear combination of equations (7) gives

$$
a y_{1} \operatorname{gcd}\left\{\alpha_{1}, \alpha_{3}\right\}+b y_{2} \operatorname{gcd}\left\{\alpha_{2}, \alpha_{3}\right\} \equiv-x_{3} \bmod \alpha_{3},
$$

so that (i) is proved. Finally, (ii) follows from (15).

In the following, we make use of (iii) in Theorem 12 in order to investigate the case of the best known classes of arithmetic discrete planes, namely the naive, the standard, and the graceful ones. Let $\mathcal{P}=\mathfrak{P}(\mathbf{v}, \mu, w)$ be an arithmetic discrete plane and let $\boldsymbol{\alpha} \in \mathbb{Z}^{3}$ such that $\langle\boldsymbol{\alpha}, \mathbf{v}\rangle=w$. We assume here that there exists $i \in\{1,2,3\}$ such that $\alpha_{i}=1$, say $\alpha_{3}=1$. Let us emphasize that, according to (11), $\Gamma_{\boldsymbol{\alpha}}=\mathbb{Z} \mathbf{e}_{\mathbf{1}}+\mathbb{Z} \mathbf{e}_{\mathbf{2}}$ in that latter case. Moreover, Theorem 12 yields a simple formula for the height of $\mathcal{P}$, that is,

$$
H_{\mathcal{P}, \boldsymbol{\alpha}}(\mathbf{y})=-\left\lfloor\frac{v_{1} y_{1}+v_{2} y_{2}+\mu}{w}\right\rfloor .
$$

One thus obtains:

Corollary 13 If $\alpha_{3}=1$, then the function $\pi_{\boldsymbol{\alpha}}^{-1}: \Gamma_{\boldsymbol{\alpha}} \rightarrow \mathcal{P}$ is defined, for all $\mathbf{y} \in \Gamma_{\boldsymbol{\alpha}}$ with $\mathbf{y}=y_{1} \mathbf{e}_{\mathbf{1}}+y_{2} \mathbf{e}_{\mathbf{2}}$, by:

$$
\pi_{\boldsymbol{\alpha}}^{-1}(\mathbf{y})=\mathbf{y}-\left\lfloor\frac{v_{1} y_{1}+v_{2} y_{2}+\mu}{w}\right\rfloor \boldsymbol{\alpha}
$$

Let us assume that $\mathbf{v} \in \mathbb{R}_{+}^{3}$ with $v_{1} \leq v_{2} \leq v_{3}$, which may be achieved by mild modifications of the coordinates axes. If $\mathcal{P}$ is either a naive or a standard discrete plane we can thus assume $\alpha_{3}=1$. If $\mathcal{P}=\mathfrak{P}(\mathbf{v}, \mu, w)$ is a graceful plane, that is, $w=\max \left(v_{1}+v_{2}, v_{3}\right)$, then it is naive if $v_{1}+v_{2} \leq v_{3}$, otherwise we may assume $\alpha_{1}=1$. Therefore, we recover the following from Corollary 13, the formula being already known in the naive case:

Corollary 14 Let $\mathcal{P}=\mathfrak{P}(\mathbf{v}, \mu, w)$ with $0 \leq v_{1} \leq v_{2} \leq v_{3}$.

If $\mathcal{P}$ is naive, then $\boldsymbol{\alpha}=\mathbf{e}_{\mathbf{3}}$ is functional for $\mathcal{P}$. For all $\mathbf{x} \in \mathcal{P}, \pi_{\boldsymbol{\alpha}}(\mathbf{x})=$ $x_{1} \mathbf{e}_{\mathbf{1}}+x_{2} \mathbf{e}_{\mathbf{2}}$ and for all $\mathbf{y}=y_{1} \mathbf{e}_{\mathbf{1}}+y_{2} \mathbf{e}_{\mathbf{2}} \in \Gamma_{\boldsymbol{\alpha}}$,

$$
\pi_{\alpha}^{-1}(\mathbf{y})=y_{1} \mathbf{e}_{\mathbf{1}}+y_{2} \mathbf{e}_{\mathbf{2}}-\left\lfloor\frac{v_{1} y_{1}+v_{2} y_{2}+\mu}{v_{3}}\right\rfloor \mathbf{e}_{\mathbf{3}} .
$$

If $\mathcal{P}$ is standard, then $\boldsymbol{\alpha}=\mathbf{e}_{\mathbf{1}}+\mathbf{e}_{\mathbf{2}}+\mathbf{e}_{\mathbf{3}}$ is functional for $\mathcal{P}$. For all $\mathbf{x} \in \mathcal{P}$, $\pi_{\boldsymbol{\alpha}}(x)=\left(x_{1}-x_{3}\right) \mathbf{e}_{\mathbf{1}}+\left(x_{2}-x_{3}\right) \mathbf{e}_{\mathbf{2}}$, and for all $\mathbf{y}=y_{1} \mathbf{e}_{\mathbf{1}}+y_{2} \mathbf{e}_{\mathbf{2}} \in \Gamma_{\boldsymbol{\alpha}}$,

$$
\pi_{\boldsymbol{\alpha}}^{-1}(\mathbf{y})=y_{1} \mathbf{e}_{\mathbf{1}}+y_{2} \mathbf{e}_{\mathbf{2}}-\left\lfloor\frac{v_{1} y_{1}+v_{2} y_{2}+\mu}{v_{1}+v_{2}+v_{3}}\right\rfloor\left(\mathbf{e}_{\mathbf{1}}+\mathbf{e}_{\mathbf{2}}+\mathbf{e}_{\mathbf{3}}\right) .
$$

If $\mathcal{P}$ is graceful with $w=v_{1}+v_{2}$, then $\boldsymbol{\alpha}=\mathbf{e}_{\mathbf{1}}+\mathbf{e}_{\mathbf{2}}$ is functional for $\mathcal{P}$. For 


$$
\begin{gathered}
\text { all } \mathbf{x} \in \mathcal{P}, \pi_{\boldsymbol{\alpha}}(x)=\left(x_{2}-x_{1}\right) \mathbf{e}_{\mathbf{2}}+x_{3} \mathbf{e}_{\mathbf{3}}, \text { and for all } \mathbf{y}=y_{2} \mathbf{e}_{\mathbf{2}}+y_{3} \mathbf{e}_{\mathbf{3}} \in \Gamma_{\boldsymbol{\alpha}}, \\
\pi_{\boldsymbol{\alpha}}^{-1}(\mathbf{y})=y_{2} \mathbf{e}_{\mathbf{2}}+y_{3} \mathbf{e}_{\mathbf{3}}-\left\lfloor\frac{v_{2} y_{2}+v_{3} y_{3}+\mu}{v_{1}+v_{2}}\right\rfloor\left(\mathbf{e}_{\mathbf{1}}+\mathbf{e}_{\mathbf{2}}\right) .
\end{gathered}
$$

\section{Local configurations and m-cubes}

\subsection{First definitions}

The aim of this section is to apply the previous results to the study of $(m, n)$ cubes and local configurations, generalizing the study performed for rational naive planes in [VC97,Sch97,Gér99,VC99,Col02]. For the sake of consistency in the notation, we call them here $\mathbf{m}$-cubes with $\mathbf{m}=\left(m_{1}, m_{2}\right)$ rather than $(m, n)$-cubes.

Let $\mathcal{P}=\mathfrak{P}(\mathbf{v}, \mu, w)$ be an arithmetic discrete plane and let $\boldsymbol{\alpha} \in \mathbb{Z}^{3}$ such that $\operatorname{gcd}(\boldsymbol{\alpha})=1$ and $\langle\boldsymbol{\alpha}, \mathbf{v}\rangle=w$ (recall that if $\mathbf{v} \in \mathbb{Z}^{3}$ and $\operatorname{gcd}(\mathbf{v})=1$, then the existence of $\boldsymbol{\alpha}$ is ensured by Proposition 9). The results in both the present and the following sections rely on Corollary 13. We shall assume that $\alpha_{3}=1$ in all that follows. Indeed, we heavily use the explicit and simple expression of the preimage of a point in $\Gamma_{\boldsymbol{\alpha}}$ given by (18) obtained under the assumption $\alpha_{3}=1$. Note that, since $w=\alpha_{1} v_{1}+\alpha_{2} v_{2}+\alpha_{3} \alpha_{3}, \alpha_{3}=1$ is equivalent to $w \in v_{1} \mathbb{Z}+v_{2} \mathbb{Z}+v_{3}$, i.e., $w-v_{3} \in \operatorname{gcd}\left(v_{1}, v_{2}\right) \mathbb{Z}$ in the rational case.

Also note that there does not always exist a functional vector $\boldsymbol{\alpha}$ with $\alpha_{3}=1$. Consider for instance the case $\mathbf{v}=(6,10,15)$ with $w=20$ : it is impossible to express $w$ as $\alpha_{1} v_{1}+\alpha_{2} v_{2}+\alpha_{3} v_{3}$ with one of the $\alpha_{i}$ 's equal to 1 .

Let $\mathbf{m} \in\left(\mathbb{N}^{\star}\right)^{2}$ be given. By $\mathbf{m}$-cube we mean a local configuration in the discrete plane that can be observed thanks to $\pi_{\boldsymbol{\alpha}}$ through an $\mathbf{m}$-window in the functional lattice $\Gamma_{\boldsymbol{\alpha}}=\mathbb{Z} \mathbf{e}_{\boldsymbol{1}}+\mathbb{Z} \mathbf{e}_{\boldsymbol{2}}$ (see Figure 2). More precisely,

Definition 15 Let $\mathbf{m} \in\left(\mathbb{N}^{\star}\right)^{2}$ and $\mathbf{y} \in \Gamma_{\boldsymbol{\alpha}}$. The $\mathbf{m}$-cube $\mathcal{C}(\mathbf{y}, \mathbf{m})$ of $\mathcal{P}$ is defined as the following subset of $\mathcal{P}$ :

$$
\mathcal{C}(\mathbf{y}, \mathbf{m})=\left\{\pi_{\boldsymbol{\alpha}}^{-1}(\mathbf{y}+\mathbf{z}), \mathbf{z} \in \llbracket 0, m_{1}-1 \rrbracket \mathbf{e}_{\mathbf{1}}+\llbracket 0, m_{2}-1 \rrbracket \mathbf{e}_{\mathbf{2}}\right\} .
$$

Two $\mathbf{m}$-cubes $\mathcal{C}$ and $\mathcal{C}^{\prime}$ are called translation equivalent if there exists a vector $\mathbf{z} \in \mathbb{Z}^{3}$ such that $\mathcal{C}^{\prime}=\mathcal{C}+\mathbf{z}$.

In order to enumerate the different types of $\mathbf{m}$-cubes that occur in $\mathcal{P}$, that is, the different equivalence classes for the translation equivalence, we represent them as local configurations as follows. Recall that $H_{\mathcal{P}, \boldsymbol{\alpha}}$ is defined in (17). 

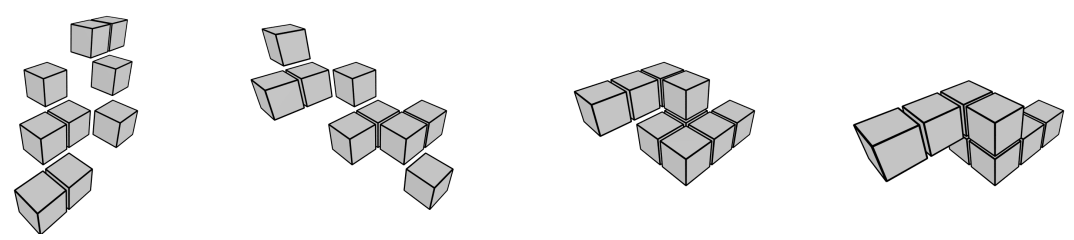

Fig. 2. From left to right: the $(3,3)$-cube of $\mathfrak{P}(\mathbf{v}, 0,9)$ (resp. $\mathfrak{P}(\mathbf{v}, 0,11), \mathfrak{P}(\mathbf{v}, 0,21)$, $\mathfrak{P}(\mathbf{v}, 0,37))$ centered on $(0,0,0)$, where $\mathbf{v}=6 \mathbf{e}_{\mathbf{1}}+10 \mathbf{e}_{\mathbf{2}}+15 \mathbf{e}_{\mathbf{3}}$, and projected along the vector $-\mathbf{e}_{1}+\mathbf{e}_{3}$ (resp. $\left.\mathbf{e}_{1}-\mathbf{e}_{2}+\mathbf{e}_{3}, \mathbf{e}_{1}+\mathbf{e}_{3}, 2 \mathbf{e}_{1}+\mathbf{e}_{2}+\mathbf{e}_{3}\right)$.

Definition 16 Let $\mathbf{m}=\left(m_{1}, m_{2}\right) \in\left(\mathbb{N}^{\star}\right)^{2}$. A $m_{1} \times m_{2}$-rectangular word $L=$ $\left[L_{i_{1}, i_{2}}\right]_{\left(i_{1}, i_{2}\right) \in \llbracket 0, m_{1}-1 \rrbracket \times \llbracket 0, m_{2}-1 \rrbracket}$ over the infinite alphabet $\mathbb{Z}$ is called an $\mathbf{m}$-local configuration of $\mathcal{P}$ if there exists $\mathbf{y} \in \mathbb{Z}^{2}$ such that:

$$
L=\left[H_{\mathcal{P}, \boldsymbol{\alpha}}(\mathbf{z})-H_{\mathcal{P}, \boldsymbol{\alpha}}(\mathbf{y})\right]_{\mathbf{z} \in \llbracket 0, m_{1}-1 \rrbracket \mathbf{e}_{\mathbf{1}}+\llbracket 0, m_{2}-1 \rrbracket \mathbf{e}_{\mathbf{2}}} .
$$

Such a local configuration (19) is denoted by $L C(\mathbf{y}, \mathbf{m})$.

Let us note that a local configuration is a plane partition. Indeed a plane partition of $N \in \mathbb{N}$ is a rectangular word $w=\left[w_{i_{1}, i_{2}}\right]_{\left(i_{1}, i_{2}\right) \in \llbracket 0, m_{1}-1 \rrbracket \times \llbracket 0, m_{2}-1 \rrbracket}$ over the infinite alphabet $\mathbb{N}$ satisfying $N=\sum_{i, j} w_{i, j}$ and, for all $i_{1} \in \llbracket 0, m_{1}-1 \rrbracket$ and $i_{2} \in \llbracket 0, m_{2}-1 \rrbracket, \max \left\{w_{i_{1}+1, i_{2}}, w_{i_{1}, i_{2}+1}\right\} \leq w_{i_{1}, i_{2}}$.

Notation 17 Let $L=\left[L_{i_{1}, i_{2}}\right]_{\left(i_{1}, i_{2}\right) \in \llbracket 0, m_{1}-1 \rrbracket \times \llbracket 0, m_{2}-1 \rrbracket}$ be a local configuration of size $m_{1} \times m_{2}$. In all that follows, the notation $L \bmod 2$ stands for the $m_{1} \times m_{2}$ rectangular word $\left[L_{i_{1}, i_{2}} \bmod 2\right]_{\left(i_{1}, i_{2}\right) \in \llbracket 0, m_{1}-1 \rrbracket \times \llbracket 0, m_{2}-1 \rrbracket}$.

Example 18 Let us consider the arithmetic discrete plane $\mathcal{P}=\mathfrak{P}(\mathbf{v}, \mu, w)$ with $\mathbf{v}=6 \mathbf{e}_{\mathbf{1}}+10 \mathbf{e}_{\mathbf{2}}+15 \mathbf{e}_{\mathbf{3}}, \mu=0$, and $w=21$. Then $\boldsymbol{\alpha}=\mathbf{e}_{\mathbf{1}}+\mathbf{e}_{\mathbf{3}}$ is a functional vector of $\mathcal{P}$. The local configuration $L C\left(\mathbf{e}_{\mathbf{1}}+\mathbf{e}_{\mathbf{2}}, 3 \mathbf{e}_{\mathbf{1}}+3 \mathbf{e}_{\mathbf{2}}\right)$ of $\mathcal{P}$ and its preimage by $\pi_{\boldsymbol{\alpha}}^{-1}$ are illustrated in Fig. 3.
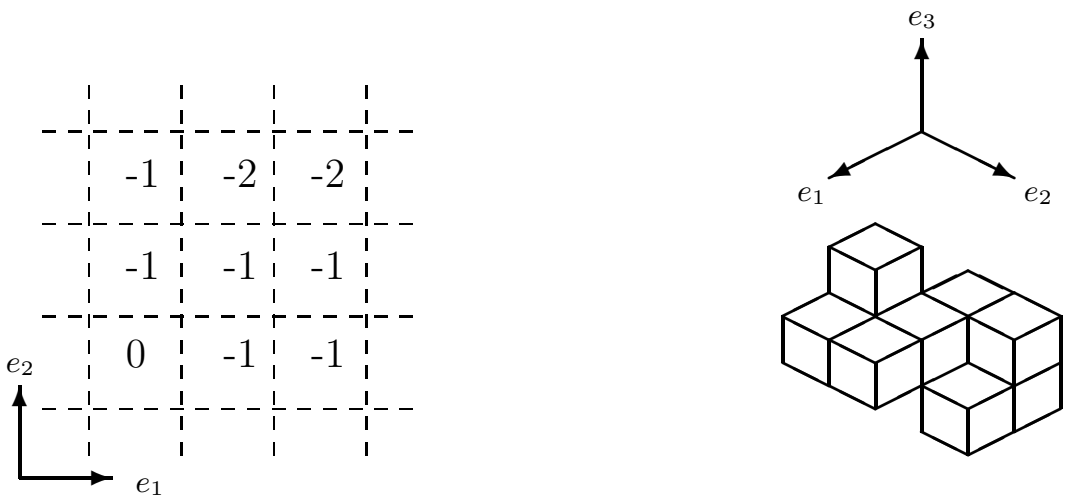

Fig. 3. From left to right: a local configuration of the discrete plane $\mathfrak{P}((6,10,15), 0,21)$ and its corresponding preimage by $\pi_{\mathbf{e}_{\mathbf{1}}+\mathbf{e}_{\mathbf{3}}}^{-1}$.

Remark 19 A local configuration is not necessarily (simply-)connected. For instance, let us consider $\mathcal{P}=\mathfrak{P}(\mathbf{v}, \mu, w)$ with $\mathbf{v}=\mathbf{e}_{\mathbf{1}}+2 \mathbf{e}_{\mathbf{2}}+7 \mathbf{e}_{\mathbf{3}}$ and $w=4$. 
Then $\boldsymbol{\alpha}=\mathbf{e}_{\mathbf{1}}-2 \mathbf{e}_{\mathbf{2}}+\mathbf{e}_{\mathbf{3}}$ is a functional vector of $\mathcal{P}$, and $L C\left(-\mathbf{e}_{\mathbf{1}}-\mathbf{e}_{\mathbf{2}}, \mathbf{e}_{\mathbf{1}}+\mathbf{e}_{\mathbf{2}}\right)$ is not connected (see Fig. 4).

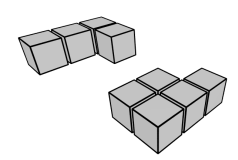

Fig. 4. The (3,3)-cube of $\mathfrak{P}(\mathbf{v}, 0,4)$ centered on $(0,0,0)$ with $\mathbf{v}=\mathbf{e}_{\mathbf{1}}+2 \mathbf{e}_{\mathbf{2}}+7 \mathbf{e}_{\mathbf{3}}$.

\subsection{A coding as a two-dimensional word}

According to [Vui99], we introduce a two-dimensional word coding in a natural way the parity of the heights $H_{\mathcal{P}, \boldsymbol{\alpha}}(\mathbf{y})$, for $\mathbf{y}$ in the lattice $\Gamma_{\boldsymbol{\alpha}}=\mathbb{Z} \mathbf{e}_{\mathbf{1}}+\mathbb{Z} \mathbf{e}_{\mathbf{2}}$. Indeed, for a naive discrete plane $\mathcal{P}$, it is well known that, given two points $\mathbf{x}$ and $\mathbf{x}^{\prime}$ of $\mathcal{P}$ such that their projections by $\pi_{\boldsymbol{\alpha}}$ are 4 -connected in the functional plane, then $\left|x_{3}-x_{3}^{\prime}\right| \leq 1$. In other words, the difference between the heights of $\mathbf{x}$ and $\mathbf{x}^{\prime}$ is at most 1 . A quite unexpected fact is that this property holds for any arithmetic discrete plane with $\alpha_{3}=1$. More precisely, it is easy to see that, for all $\mathbf{y} \in \Gamma_{\boldsymbol{\alpha}}$ and $i=1,2, H_{\mathcal{P}, \boldsymbol{\alpha}}\left(\mathbf{y}+\mathbf{e}_{\mathbf{i}}\right)-H_{\mathcal{P}, \boldsymbol{\alpha}}(\mathbf{y})$ takes only two values, namely $-\left\lfloor v_{i} / w\right\rfloor$ and $-\left\lfloor v_{i} / w\right\rfloor-1$. In each case, one of these values is odd, whereas the other one is even; we define $E_{1}$ and $O_{1}$ to be respectively the even and the odd value taken by $-\left\lfloor v_{1} / w\right\rfloor$ and $-\left\lfloor v_{1} / w\right\rfloor-1$; we similarly define $E_{2}$ and $\mathrm{O}_{2}$. It is now natural to introduce the following two-dimensional word of parity of heights by identifying $\Gamma_{\boldsymbol{\alpha}}$ to $\mathbb{Z}^{2}$ :

$$
U=\left(U_{i_{1}, i_{2}}\right)_{\left(i_{1}, i_{2}\right) \in \mathbb{Z}^{2}}=\left(H_{\mathcal{P}, \boldsymbol{\alpha}}(\mathbf{y}) \bmod 2\right)_{\mathbf{y} \in \mathbb{Z}^{2}} \in\{0,1\}^{\mathbb{Z}^{2}}
$$

Lemma 20 The two-dimensional word $U$ satisfies, for each $\left(i_{1}, i_{2}\right) \in \mathbb{Z}^{2}$

$$
U_{i_{1}, i_{2}}=0 \text { if, and only if, } v_{1} i_{1}+v_{2} i_{2}+\mu \bmod 2 w \in[0, w[\text {. }
$$

Proof. According to (17), $U_{i_{1}, i_{2}}=0$ if, and only if, $\left\lfloor\frac{v_{1} i_{1}+v_{2} i_{2}+\mu}{w}\right\rfloor$ is even, that is, $v_{1} i_{1}+v_{2} i_{2}+\mu \bmod 2 w \in[0, w[$.

The word $U$ is a two-dimensional Rote word; one-dimensional Rote words have been introduced in [Rot94]; they are defined as the infinite words over the alphabet $\{0,1\}$ that have exactly $2 n$ factors of length $n$ for every positive integer $n$, and whose set of factors is closed under complementation, i.e., every word obtained by interchanging zeros and ones in a factor of the infinite word $u$ is still a factor of $u$; two-dimensional Rote words have been studied for instance in [Vui99,BV01]. 
Definition 21 Let $W=\left[w_{i_{1}, i_{2}}\right]_{\left(i_{1}, i_{2}\right) \in \llbracket 0, m_{1}-1 \rrbracket \times \llbracket 0, m_{2}-1 \rrbracket}$ be a rectangular word of size $m_{1} \times m_{2}$ over $\{0,1\}$. We define the complement $\bar{W}$ of $W$ as follows:

$$
\bar{W}=\left[\overline{w_{i_{1}, i_{2}}}\right]_{\left(i_{1}, i_{2}\right) \in \llbracket 0, m_{1}-1 \rrbracket \times \llbracket 0, m_{2}-1 \rrbracket}, \quad \text { where } \overline{1}=0 \text { and } \overline{0}=1 \text {. }
$$

We introduce the following equivalence relation defined on the set of rectangular factors of $U$ of a given size:

$$
V \sim W \text { if, and only if, } V \in\{W, \bar{W}\} .
$$

We have the following theorem, inspired by [Vui99] where it is stated under the assumption $\operatorname{dim}_{\mathbb{Q}} v=3$ :

Theorem 22 Let $\mathcal{P}=\mathfrak{P}(\mathbf{v}, \mu, w)$ be a discrete plane that admits a functional vector $\boldsymbol{\alpha}$ satisfying $\alpha_{3}=1$. There is a natural bijection between the equivalence classes of the relation $\sim$ on the rectangular factors of the two-dimensional word $U$ of size $\mathbf{m}=\left(m_{1}, m_{2}\right)$ and the $\mathbf{m}$-local configurations of $\mathcal{P}$. Furthermore, the $\mathbf{m}$-local configurations of $\mathcal{P}$ are in one-to-one correspondence with the translation equivalence classes of $\mathbf{m}$-cubes of $\mathcal{P}$.

Proof. Let $\mathbf{m}=\left(m_{1}, m_{2}\right) \in\left(\mathbb{N}^{\star}\right)^{2}$. Consider the local configuration $L=$ $L C(\mathbf{y}, \mathbf{m})$ with the notation of Definition 16 ; one has either $L \bmod 2$ or $\overline{L \bmod 2}$ factor of the two-dimensional word $U$. Indeed if $H_{\mathcal{P}, \boldsymbol{\alpha}}(\mathbf{y})$ is even, then $L \bmod 2$ is a factor of the two-dimensional word $U$; otherwise, $H_{\mathcal{P}, \boldsymbol{\alpha}}(\mathbf{y})$ is odd and $\overline{L \bmod 2}$ is a factor of $U$.

Conversely, let us show how to reconstruct an $\mathbf{m}$-local configuration $L$ from a given $m_{1} \times m_{2}$-factor $W$ of $U$ such that $L \bmod 2$ is either $W$ or $\bar{W}$.

Let us first assume that $w_{0,0}=0$. We define a plane partition $H$ by induction: we set $H(0)=0$; let $\left(i_{1}, i_{2}\right) \in \llbracket 0, m_{1}-1 \rrbracket \times \llbracket 0, m_{2}-1 \rrbracket$. If $w_{i_{1}+1, i_{2}}=w_{i_{1}, i_{2}}$, then we set $H_{i_{1}+1, i_{2}}=H_{i_{1}, i_{2}}+E_{1}$. Otherwise, we set $H_{i_{1}+1, i_{2}}=H_{i_{1}, i_{2}}+O_{1}$. Similarly, if $w_{i_{1}, i_{2}+1}=w_{i_{1}, i_{2}}$, we set $H_{i_{1}, i_{2}+1}=H_{i_{1}, i_{2}}+E_{2}$. Otherwise, we set $H_{i_{1}, i_{2}+1}=H_{i_{1}, i_{2}}+\mathrm{O}_{2}$.

The plane partition $H$ is a local configuration of $\mathcal{P}$; indeed, if $W$ occurs at index $\left(k_{1}, k_{2}\right)$ in $U$, then $H=L C(\mathbf{y}, \mathbf{m})$ with $\mathbf{y}=k_{1} \mathbf{e}_{\mathbf{1}}+k_{2} \mathbf{e}_{\mathbf{2}}$, and $W=(H \bmod 2)$ since $H(\mathbf{y})$ is even (we have $\left.w_{0,0}=0\right)$.

Now, if $w_{0,0}=1$ we apply the same reconstruction process to $\bar{W}$. We recover again a local configuration $L C(\mathbf{y}, \mathbf{m})$ such that $\bar{W}=(L C(\mathbf{y}, \mathbf{m}) \bmod 2)$, which ends the proof of the existence of a one-to-one correspondence between equivalence classes of $\sim$ and $\mathbf{m}$-local configurations.

It is immediate to associate an $\mathbf{m}$-local configuration to an $\mathbf{m}$-cube by considering the third coordinate of the points of the $\mathbf{m}$-cube. Conversely, given a local configuration $L=\left[L_{i_{1}, i_{2}}\right]$ of $\mathcal{P}$ of size $m_{1} \times m_{2}$, we can associate to it the following subset of $\mathbb{Z}^{3}:\left\{i_{1} \mathbf{e}_{\mathbf{1}}+i_{2} \mathbf{e}_{\mathbf{2}}+L_{i_{1}, i_{2}} \mathbf{e}_{\mathbf{3}},\left(i_{1}, i_{2}\right) \in \llbracket 0, m_{1}-1 \rrbracket \times \llbracket 0, m_{2}-1 \rrbracket\right\}$. It remains to note that there exists $\mathbf{z} \in \mathbb{Z}^{3}$ such that $\mathbf{z}+\left\{i_{1} \mathbf{e}_{\mathbf{1}}+i_{2} \mathbf{e}_{\mathbf{2}}+\right.$ $\left.L_{i_{1}, i_{2}} \mathbf{e}_{\mathbf{3}}, \quad\left(i_{1}, i_{2}\right) \in \llbracket 0, m_{1}-1 \rrbracket \times \llbracket 0, m_{2}-1 \rrbracket\right\} \subset \mathcal{P}$, to conclude the proof. 


\subsection{Basic properties of the two-dimensional word $U$}

This section is devoted to the study of combinatorial properties of the twodimensional word $U$ from which we will deduce geometric properties of the local configurations and $\mathbf{m}$-cubes.

The two-dimensional word $U$ is called periodic if there exists a non-zero vector $\mathbf{z} \in \mathbb{Z}^{2}$, called period, such that $U_{\mathbf{y}+\mathbf{z}}=U_{\mathbf{y}}$ for every $\mathbf{y} \in \Gamma_{\boldsymbol{\alpha}}$. The set of its periods is a lattice whose rank is determined by the dimension over $\mathbb{Q}$ of the vector space generated by the coordinates of the vector $\mathbf{v}$ :

Proposition 23 The rank of the lattice of periods of the two-dimensional word $U$ is $3-\operatorname{dim}_{\mathbb{Q}} v$.

Proof. The proposition can easily be deduced from the following observation: for a non-zero element $\left(\ell_{1}, \ell_{2}\right) \in \mathbb{Z}^{2}, \frac{v_{1} \ell_{1}+v_{2} \ell_{2}}{w} \in \mathbb{Q}$ if, and only if, there exists a non-zero integer $k$ such that $\left(k v_{1}, k v_{2}\right)$ is a period of $U$. Let us prove this latter statement. If $\frac{v_{1} \ell_{1}+v_{2} \ell_{2}}{w} \in \mathbb{Q}$, then there exists a non-zero integer $k$ such that $v_{1}\left(k \ell_{1}\right)+v_{2}\left(k \ell_{2}\right) \in 2 w \mathbb{Z}$, hence $\left(k \ell_{1}, k \ell_{2}\right)$ is a period. Otherwise, if $\frac{v_{1} \ell_{1}+v_{2} \ell_{2}}{w} \notin$ $\mathbb{Q}$, then the density of $\left(v_{1}\left(k \ell_{1}\right)+v_{2}\left(k \ell_{2}\right) \bmod 2 w\right)_{k \in \mathbb{Z}}$ yields the desired result.

A key ingredient in the combinatorial study of the two-dimensional word $U$ is the following lemma. This is a standard approach in symbolic dynamics for the study of sturmian words [PF02], and more generally, of infinite words coding rotations as well as double rotations [BV00,BV01] in the torus $\mathbb{R} / \mathbb{Z}$. This lemma will allow us in Sections 5.4 and 5.5 to derive enumeration as well as statistical properties for the factors of $U$, and thus for $\mathbf{m}$-cubes of $\mathcal{P}$.

In all that follows intervals are considered as intervals of the torus $\mathbb{R} / 2 w \mathbb{Z}$.

Lemma 24 Let $W=\left[w_{i_{1}, i_{2}}\right]_{\left(i_{1}, i_{2}\right) \in \llbracket 0, m_{1}-1 \rrbracket \times \llbracket 0, m_{2}-1 \rrbracket}$ be a rectangular word of size $m_{1} \times m_{2}$ over $\{0,1\}$. Let $I_{0}=\left[0, w\left[\right.\right.$ and $I_{1}=[w, 2 w[$. Let

$$
I_{W}=\bigcap_{i_{1}=0}^{m_{1}-1} \bigcap_{i_{2}=0}^{m_{2}-1}\left(I_{w_{i_{1}, i_{2}}}-\left(v_{1} i_{1}+v_{2} i_{2}\right) \bmod 2 w\right) .
$$

The set $I_{W}$ is a left-closed right-open interval of $[0,2 w[$.

Let $\mathcal{P}=\mathfrak{P}(\mathbf{v}, \mu, w)$ be a discrete plane with $w-v_{3} \in v_{1} \mathbb{Z}+v_{2} \mathbb{Z}$. If $\operatorname{dim}_{\mathbb{Q}} \mathbf{v}>1$ or $\mathcal{P}$ is rational and $\operatorname{gcd}\left(v_{1}, v_{2}, 2 w\right)=1$, then a rectangular word $W$ over $\{0,1\}$ is a factor of $U$ if, and only if, $I_{W} \neq \emptyset$. Otherwise, if $\mathcal{P}$ is rational and $\operatorname{gcd}\left(v_{1}, v_{2}, 2 w\right)=2$, then a rectangular word $W$ over $\{0,1\}$ is a factor of $U$ if, and only if, $I_{W}$ contains an integer with the same parity as $\mu$.

Proof. The proof is inspired by [BV00] where more details are given. It is easily shown that the sets $I_{W}$ consist of finite unions of left-closed right-open 
intervals. We then show by induction that each set $I_{W}$ is an interval. Indeed this can be deduced from the following remark: if $I$ and $J$ are two left-closed right-open intervals of $\mathbb{R} / \mathbb{Z}$ whose intersection is non-connected, then the sum of their lengths is strictly larger than 1 .

Let $W=\left[w_{i_{1}, i_{2}}\right]_{\left(i_{1}, i_{2}\right) \in \llbracket 0, m_{1}-1 \rrbracket \times \llbracket 0, m_{2}-1 \rrbracket}$ be a rectangular word of size $m_{1} \times m_{2}$ over $\{0,1\}$. One first checks that $W$ occurs in the sequence $U$ at index $\left(k_{1}, k_{2}\right)$ if, and only if, $v_{1} k_{1}+v_{2} k_{2}+\mu \in I_{W}$. Indeed, $W$ occurs in the sequence $U$ at index $\left(k_{1}, k_{2}\right)$ if, and only if, $\forall\left(i_{1}, i_{2}\right) \in \llbracket 0, m_{1}-1 \rrbracket \times \llbracket 0, m_{2}-1 \rrbracket, w_{i_{1}, i_{2}}=$ $U_{k_{1}+i_{1}, k_{2}+i_{2}}$, that is, $v_{1}\left(k_{1}+i_{1}\right)+v_{2}\left(k_{2}+i_{2}\right)+\mu \bmod 2 w \in I_{w_{i_{1}, i_{2}}}$, or else, $v_{1} k_{1}+v_{2} k_{2}+\mu \bmod 2 w \in I_{w_{i_{1}, i_{2}}}-\left(v_{1} i_{1}+v_{2} i_{2}\right)$.

Conversely, let $W$ be a rectangular word over $\{0,1\}$ such that $I_{W} \neq \emptyset$.

If $\operatorname{dim}_{\mathbb{Q}} \mathbf{v}>1$, then the density of $\left(v_{1} k_{1}+v_{2} k_{2}+\mu \bmod 2 w\right)_{\left(k_{1}, k_{2}\right) \in \mathbb{Z}^{2}}$ in $[0,2 w[$ and the fact that $I_{W}$ has non-empty interior imply that there exists $\left(k_{1}, k_{2}\right)$ such that $v_{1} k_{1}+v_{2} k_{2}+\mu \in I_{W}$, hence $W$ occurs in $U$ at index $\left(k_{1}, k_{2}\right)$.

Next assume $\mathcal{P}$ is rational, that is, $\operatorname{dim}_{\mathbb{Q}} \mathbf{v}=1$. One has $\operatorname{gcd}\left\{v_{1}, v_{2}, 2 w\right\} \in$ $\{1,2\}$ since $w=\alpha_{1} v_{1}+\alpha_{2} v_{2}+v_{3}$ and $\operatorname{gcd}(v)=1$. Let us note that if $\operatorname{gcd}\left\{v_{1}, v_{2}, 2 w\right\}=2$, then $w$ is odd since $\operatorname{gcd}\left(v_{1}, v_{2}, w\right)=1$.

Since the sets $I_{W}$ are semi-open intervals of integer lengths, then they are nonempty as soon as they contain an integer point. Let $k \in I_{W}$. If $\operatorname{gcd}\left\{v_{1}, v_{2}, 2 v_{3}\right\}=$ 1 , then there exists $\left(k_{1}, k_{2}\right)$ such that $v_{1} k_{1}+v_{2} k_{2}+\mu \equiv k \bmod 2 w$. Assume $\operatorname{gcd}\left\{v_{1}, v_{2}, 2 v_{3}\right\}=2$. Then for every $\left(k_{1}, k_{2}\right), v_{1} k_{1}+v_{2} k_{2}+\mu \bmod 2 w$ has the same parity as $\mu$. Hence there exists $\left(k_{1}, k_{2}\right)$ such that $v_{1} k_{1}+v_{2} k_{2}+\mu \equiv k$ $\bmod 2 w$ if $k$ has the same parity as $\mu$.

Let us recall [Vui99] that the set of factors of the two-dimensional word $U$ is closed under complementation (see Definition 21) under the assumption $\operatorname{dim}_{\mathbb{Q}} v=3$. This still holds true if $\operatorname{dim}_{\mathbb{Q}}>1$. But in the rational case one has to be more cautious:

Proposition 25 Let $W$ be a rectangular factor of $U$. One has $I_{\bar{W}}=I_{W}+w$. Let $\mathcal{P}=\mathfrak{P}(\mathbf{v}, \mu, w)$ be a discrete plane with $w-v_{3} \in v_{1} \mathbb{Z}+v_{2} \mathbb{Z}$.

If either $\operatorname{dim}_{\mathbb{Q}}\left(v_{1}, v_{2}, w\right) \geq 2$ or if $\mathcal{P}$ is rational and $\operatorname{gcd}\left(v_{1}, v_{2}, 2 w\right)=1$, then the language of $U$ is closed under complementation.

Otherwise, assume that $\mathcal{P}$ is rational and $\operatorname{gcd}\left(v_{1}, v_{2}, 2 w\right)=2$. Let $W$ be a rectangular factor of the two-dimensional word $U$. Then $\bar{W}$ is a factor of $U$ if, and only if, $I_{W}$ contains both an even and an odd integer.

Proof. One easily checks from the definition of $I_{W}$ that $I_{\bar{W}}=I_{W}+w$. Hence $I_{W} \neq \emptyset$ if, and only if, $I_{\bar{W}} \neq \emptyset$. Furthermore, in the rational case, when both intervals are non-empty, then $I_{W}$ and $I_{\bar{W}}$ contain points with different parities, since $w$ is odd. We thus conclude thanks to Lemma 24.

Corollary 26 A rectangular word $L$ over the infinite alphabet $\mathbb{Z}$ occurs as a local configuration of $\mathcal{P}$ if, and only if, $I_{L} \bmod _{2} \neq \emptyset$. 
Proof. If the rectangular factor $L$ occurs in $\mathcal{P}$, then $I_{L \bmod { }_{2}} \neq \emptyset$, according to Lemma 24. Conversely, let $L$ be rectangular factor such that $I_{L \bmod { }_{2}} \neq \emptyset$. We set $W=L \bmod 2$. According to Theorem 22, it is sufficient to prove that either $W$ or $\bar{W}$ is a factor of $U$. In the irrational case or in the rational case under the assumption $\operatorname{gcd}\left(v_{1}, v_{2}, 2 w\right)=1$, then $I_{W} \neq \emptyset$ implies $W$ is factor of $U$, by Lemma 24 . In the rational case with $\operatorname{gcd}\left(v_{1}, v_{2}, 2 w\right)=1$, one concludes by using $I_{\bar{W}}=I_{W}+w$ and by noticing that $w$ is odd.

\subsection{Enumeration of local configurations}

Let us now investigate the enumeration of $\mathbf{m}$-cubes $\left(\mathbf{m}=\left(m_{1}, m_{2}\right)\right)$ occuring in a given arithmetic plane. The number of $(3,3)$-cubes included in a given rational naive arithmetic discrete plane has been proved to be at most 9 in [VC97]. More generally, in [Rev95,Gér99], the authors proved that, given a rational naive arithmetic discrete plane $\mathcal{P}, \mathcal{P}$ contains at most $m_{1} m_{2} \mathbf{m}$-cubes (to be more precise, translation equivalence classes of $\mathbf{m}$-cubes). In [Gér99] local configurations which are non-necessarily rectangular are also considered. In the following theorem, we show that this property also holds in our framework. For the sake of simplicity, we omit to mention that we consider translation equivalence classes of $\mathbf{m}$-cubes:

Theorem 27 Let $\mathcal{P}=\mathfrak{P}(\mathbf{v}, \mu, w)$ be a discrete plane with $w-v_{3} \in v_{1} \mathbb{Z}+v_{2} \mathbb{Z}$. Let $\mathbf{m}=\left(m_{1}, m_{2}\right) \in\left(\mathbb{N}^{\star}\right)^{2}$. Then, $\mathcal{P}$ contains at most $m_{1} m_{2} \mathbf{m}$-cubes. More precisely, one has:

(1) If $\operatorname{dim}_{\mathbb{Q}} \mathbf{v}=1, \mathbf{v} \in \mathbb{Z}^{3}, \mu \in \mathbb{Z}, w \in \mathbb{Z}$ and $\operatorname{gcd}(\mathbf{v})=1$, then $\mathcal{P}$ contains at most $w \mathbf{m}$-cubes for every $\mathbf{m}=\left(m_{1}, m_{2}\right) \in\left(\mathbb{N}^{\star}\right)^{2}$. Moreover, for $m_{1}$ and $m_{2}$ large enough, $\mathcal{P}$ contains exactly $w \mathbf{m}$-cubes.

(2) Let us assume $\operatorname{dim}_{\mathbb{Q}} \mathbf{v}=2$. Let $\left(p_{1}, p_{2}\right) \in \mathbb{Z}^{2}$ be a generator of the lattice of periods of the two-dimensional word $U$. Then $\mathcal{P}$ contains at most $m_{1}\left|p_{2}\right|+$ $m_{2}\left|p_{1}\right|-\min \left\{m_{1},\left|p_{1}\right|\right\} \min \left\{m_{2},\left|p_{2}\right|\right\} \mathbf{m}$-cubes for $\left(m_{1}, m_{2}\right) \in \mathbb{N}^{2}$.

(3) If $\operatorname{dim}_{\mathbb{Q}} \mathbf{v}=3$, then $\mathcal{P}$ contains exactly $m_{1} m_{2} \mathbf{m}$-cubes for every $\mathbf{m}=$ $\left(m_{1}, m_{2}\right) \in\left(\mathbb{N}^{\star}\right)^{2}$.

Let us note that the bounds upon which the previous results hold for $m_{1}$ and $m_{2}$ can be explicitly computed in terms of $\mathbf{v}$ and $w$.

Proof. According to Lemma 24 and Corollary 26, it amounts to count the number of intervals $I_{W}$. The extremal points of the intervals $I_{W}$ for $W$ factor of size $m_{1} \times m_{2}$ of the sequence $U$ belong to $-\left(i_{1} v_{1}+i_{2} v_{2}\right)$ and $-\left(i_{1} v_{1}+i_{2} v_{2}\right)+w$, for $0 \leq i_{1} \leq m_{1}-1$ and $0 \leq i_{2} \leq m_{2}-1$. There are at most $2 m_{1} m_{2}$ such points. The upper bound $m_{1} m_{2}$ thus follows from Theorem 22 .

(1) Assume $\operatorname{dim}_{\mathbb{Q}} \mathbf{v}=1$. Then the extremal points of the intervals $I_{W}$ are among the integers $\{0,1, \ldots, 2 w-1\}$ and the bound $w$ follows from Theo- 
rem 22. To state the second assertion, it remains to show that each point of the set $\{0,1, \ldots, 2 w-1\}$ can be expressed as $-\left(i_{1} v_{1}+i_{2} v_{2}\right) \bmod 2 w$ or $-\left(i_{1} v_{1}+i_{2} v_{2}\right)+w \bmod 2 w$, with $\left(i_{1}, i_{2}\right) \in\left(\mathbb{N}^{\star}\right)^{2}$. If $\operatorname{gcd}\left(v_{1}, v_{2}, 2 w\right)=$ 1 , the statement follows from Bezout's Lemma. Let us assume now that $\operatorname{gcd}\left(v_{1}, v_{2}, 2 w\right)=2$. Then $w$ is odd and the integers $-\left(i_{1} v_{1}+i_{2} v_{2}\right) \bmod 2 w$, for all $\left(i_{1}, i_{2}\right) \in\left(\mathbb{N}^{\star}\right)^{2}$, are the even elements of $\{0,1, \ldots, 2 w-1\}$ while the integers $-\left(i_{1} v_{1}+i_{2} v_{2}\right)+w \bmod 2 w$, for all $\left(i_{1}, i_{2}\right) \in\left(\mathbb{N}^{\star}\right)^{2}$, are the odd ones. We then apply Proposition 25.

(2) Assume that $\operatorname{dim}_{\mathbb{Q}} \mathbf{v}=2$. According to Proposition 23, the lattice of periods of $U$ has dimension 1 . Let $\left(p_{1}, p_{2}\right)$ be a generator of this lattice. One checks that there are at most $2 m_{1}\left|p_{2}\right|+2 m_{2}\left|p_{1}\right|-2 \min \left\{m_{1},\left|p_{1}\right|\right\} \min \left\{m_{2},\left|p_{2}\right|\right\}$ factors of $U$ of size $m_{1} \times m_{2}$. We then apply Proposition 25 .

(3) Assume now $\operatorname{dim}_{\mathbb{Q}} \mathbf{v}=3$. Then there are exactly $2 m_{1} m_{2}$ points of the form $-\left(i_{1} v_{1}+i_{2} v_{2}\right)$ and $-\left(i_{1} v_{1}+i_{2} v_{2}\right)+w$, hence $2 m_{1} m_{2}$ rectangular factors of $U$ of size $m_{1} \times m_{2}$. We then apply Proposition 25 .

\subsection{Statistical properties}

The frequency of occurrence of a rectangular word $W$ in $U$ is defined as the limit, if it exists, of the number of occurrences of $W$ in the central square factor of $U$ :

$$
f(W)=\lim _{n \rightarrow \infty} \frac{\operatorname{Card}\left\{\left(k_{1}, k_{2}\right) \in \llbracket-n, n \rrbracket^{2}, W \text { occurs at index }\left(k_{1}, k_{2}\right) \text { in } U\right\}}{(2 n+1)^{2}} .
$$

The frequency of occurrence of a translation equivalence class of an $\mathbf{m}$-cube $\mathcal{C}$ in $\mathcal{P}$ is defined as the limit, if it exists, of the number of occurrences of integer translates of $C$ in the central square pattern of $\mathcal{P}$ :

$$
\begin{gathered}
f(\mathcal{C})=\lim _{n \rightarrow \infty} \frac{1}{(2 n+1)^{2}} \cdot \operatorname{Card}\left\{\mathbf{y} \in \llbracket-n, n \rrbracket \mathbf{e}_{\mathbf{1}}+\llbracket-n, n \rrbracket \mathbf{e}_{\mathbf{2}}, \pi_{\boldsymbol{\alpha}}^{-1}(\mathbf{y}+\mathbf{z})=\mathcal{C},\right. \\
\left.\qquad \text { for } \mathbf{z} \in \llbracket 0, m_{1}-1 \rrbracket \mathbf{e}_{\mathbf{1}}+\llbracket 0, m_{2}-1 \rrbracket \mathbf{e}_{\mathbf{2}}\right\} .
\end{gathered}
$$

We prove below that the frequencies of occurrence of rectangular factors in $U$ do exist, and that they have a simple expression in terms of the lengths of the intervals $I_{W}$. We thus recover the corresponding result for $\mathbf{m}$-cubes. This gives us a simple algorithmic way of computing these frequencies.

Theorem 28 Let $\mathfrak{P}(\mathbf{v}, \mu, w)$ be a discrete plane such that $w-v_{3} \in \operatorname{gcd}\left(v_{1}, v_{2}\right) \mathbb{Z}$. Let $\mathcal{C}$ be an $\mathbf{m}$-cube. Let $L$ be the corresponding local configuration. We set $W=L \bmod 2$. Then the frequency $f(\mathcal{C})$ exists and satisfies:

$$
f(\mathcal{C})=\frac{\left|I_{W}\right|}{w}
$$


Proof. Let $\mathcal{C}$ be an $\mathbf{m}$-cube of $\mathcal{P}$. Let $L$ be the corresponding local configuration. We set $W=L \bmod 2$. According to Theorem 22 and Lemma 24, the cardinality in (21) also reads

$$
\text { Card }\left\{v_{1} k_{1}+v_{2} k_{2}+\mu \in I_{W} \cup I_{\bar{W}},\left(k_{1}, k_{2}\right) \in \llbracket-n, n \rrbracket^{2}\right\}
$$

Indeed $I_{W}=I_{\bar{W}}+w$, by Proposition 25, hence $I_{W} \cap I_{\bar{W}}=\emptyset$.

Let us first assume that $\operatorname{dim}_{\mathbb{Q}} \mathbf{v} \geq 2$. The sequence $(n \alpha)_{n \in \mathbb{Z}}$ for $\alpha$ irrational is well-distributed, that is, given an interval $I$ of $\mathbb{R} / \mathbb{Z}$,

$$
\lim _{n \rightarrow \infty} \frac{\operatorname{Card}\{-n \leq i \leq n, i \alpha+\gamma \in I\}}{2 n+1}=|I|
$$

uniformly in $\gamma[\mathrm{KN} 85]$; hence $f(W)=\frac{I_{W}}{2 w}=f(\bar{W})$. We deduce that $f(\mathcal{C})=$ $f(W)+f(\bar{W})=2 \frac{\left|I_{W}\right|}{2 w}=\frac{\left|I_{W}\right|}{w}$.

Let us next assume that $\operatorname{dim}_{\mathbb{Q}} \mathbf{v}=1$.

If $\operatorname{gcd}\left\{v_{1}, v_{2}, 2 w\right\}=1$, then one checks that for every $k \in[0,2 w[$,

$$
\lim _{n \rightarrow \infty} \frac{\operatorname{Card}\left\{\left(k_{1}, k_{2}\right) \in \llbracket-n, n \rrbracket^{2}, v_{1} k_{1}+v_{2} k_{2}+\mu \equiv k \bmod 2 w\right\}}{(2 n+1)^{2}}=\frac{1}{2 w}
$$

Hence $f(W)=\frac{I_{W}}{2 w}=f(\bar{W})$, and the result follows.

If $\operatorname{gcd}\left\{v_{1}, v_{2}, 2 w\right\}=2$, let us recall that this implies that $w$ is odd. Then one checks that for every integer $k \in[0,2 w$ [ with the same parity as $\mu$,

$$
\lim _{n \rightarrow \infty} \frac{\operatorname{Card}\left\{\left(k_{1}, k_{2}\right) \in \llbracket-n, n \rrbracket^{2}, v_{1} k_{1}+v_{2} k_{2}+\mu \equiv k \bmod 2 w\right\}}{(2 n+1)^{2}}=\frac{1}{w},
$$

otherwise, $\operatorname{Card}\left\{\left(k_{1}, k_{2}\right) \in \llbracket-n, n \rrbracket^{2}, v_{1} k_{1}+v_{2} k_{2}+\mu \equiv k \bmod 2 w\right\}=0$ for every $n$. Hence $f(W)$ (resp. $f(\bar{W})$ ) is equal to the number of integers in $I_{W}$ with the same parity (resp. with a different parity) as $\mu$ divided by $w$. Furthermore, an integer $k \in \mathbb{Z}$ with the same parity as $\mu$ belongs to $I_{W}$ if, and only if, the integer $k+w$ (which has a different parity) belongs to $I_{\bar{W}}$. One has $f(C)=f(W)+f(\bar{W})$. This implies that $f(C)$ is equal to the total number of integers of $I_{W}$ (or equivalently of $I_{\bar{W}}$ ) divided by $w$, which ends the proof. We thus get $f(\mathcal{C})=\frac{I_{W}}{w}$.

Remark 29 The same approach can be used to study stability by centrosymmetry of local configurations (see Figure 5), in the flavour of [BV01], where the set of factors of $U$ is proved to be closed under centrosymmetry if $\operatorname{dim}_{\mathbb{Q}}(\mathbf{v})=3$. We can similarly prove that if $\mathfrak{P}(\mathbf{v}, \mu, w)$ is a discrete plane such that $w-v_{3} \in$ $\operatorname{gcd}\left(v_{1}, v_{2}\right)$, then the set of translation equivalence classes of $\mathbf{m}$-cubes is closed under centrosymmetry. Furthermore, centrosymmetric $\mathbf{m}$-cubes have the same frequency. 

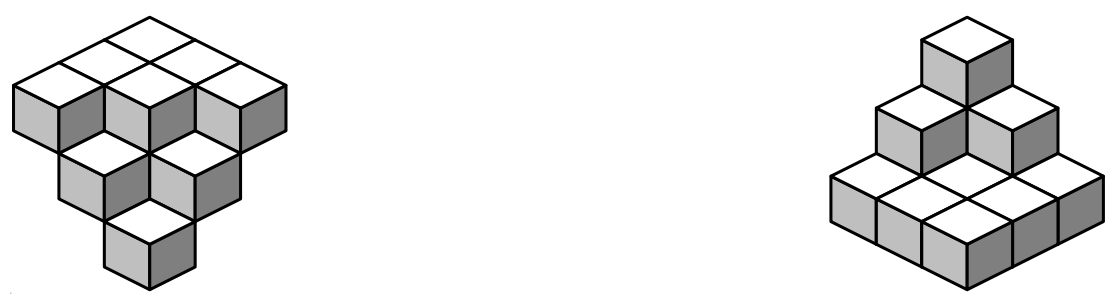

Fig. 5. From left to right: A (3,3)-cube and its centrosymmetric image.

\section{From functional arithmetic discrete planes to naive ones}

Let $\mathcal{P}=\mathfrak{P}(\mathbf{v}, \mu, w)$ be a rational arithmetic discrete plane with $\mathbf{v} \in \mathbb{N}^{3}$, $\mu \in \mathbb{Z}$ and $w \in \mathbb{N}^{\star}$. Recall that such an assumption is not restrictive since the isometry group of the unit cube $[-0.5,0.5]^{3}$ acts on the set of arithmetic discrete planes.

We assume furthermore that there exists a functional vector $\boldsymbol{\alpha} \in \mathbb{Z}^{3}$ of $\mathcal{P}$ satisfying $\alpha_{3}=1$ and that $w \geq \max \left\{v_{1}, v_{2}\right\}$. The aim of this section is to show that, under these hypotheses, $\mathcal{P}$ can be considered as a naive plane up to a change of basis in $\mathbb{Z}^{3}$. We then discuss two applications.

Lemma 30 Let $\mathcal{P}=\mathfrak{P}(\mathbf{v}, \mu, w)$ be a rational arithmetic discrete plane with $\mathbf{v} \in \mathbb{N}^{3}, \mu \in \mathbb{Z}$ and $w \in \mathbb{N}^{\star}$ and such that $w \geq \max \left\{v_{1}, v_{2}\right\}$. Let $\boldsymbol{\alpha} \in \mathbb{Z}^{3}$ be a functional vector of $\mathcal{P}$. If $\alpha_{3}=1$ then $\mathcal{P}$ is a naive discrete plane in $\mathbb{Z}^{3}$ endowed with the basis $\left\{\mathbf{e}_{\mathbf{1}}, \mathbf{e}_{\mathbf{2}}, \boldsymbol{\alpha}\right\}$.

Proof. Let $\mathbf{x}=\left(x_{1}, x_{2}, x_{3}\right) \in \mathbb{Z}^{3}$ and let $\mathbf{y}=\pi_{\boldsymbol{\alpha}}(\mathbf{x})=y_{1} \mathbf{e}_{\mathbf{1}}+y_{2} \mathbf{e}_{\mathbf{2}} \in \Gamma_{\boldsymbol{\alpha}}$. According to Corollary 13, one has $x_{1}=y_{1}+H_{\mathcal{P}, \boldsymbol{\alpha}}(\mathbf{y}) \alpha_{1}, x_{2}=y_{2}+H_{\mathcal{P}, \boldsymbol{\alpha}}(\mathbf{y}) \alpha_{2}$ and $x_{3}=H_{\mathcal{P}, \boldsymbol{\alpha}}(\mathbf{y})$ with $H_{\mathcal{P}, \boldsymbol{\alpha}}(\mathbf{y})=-\left\lfloor\frac{v_{1} y_{1}+v_{2} y_{2}+\mu}{w}\right\rfloor$. One then checks that $0 \leq v_{1} x_{1}+v_{2} x_{2}+v_{3} x_{3}+\mu<w$ if, and only if, $0 \leq v_{1} y_{1}+v_{2} y_{2}+w x_{3}+\mu<w$. Hence,

$$
\mathbf{x} \in \mathcal{P} \Longleftrightarrow 0 \leq v_{1} y_{1}+v_{2} y_{2}+w x_{3}+\mu<w
$$

which yields the result since $x_{1} \mathbf{e}_{1}+x_{2} \mathbf{e}_{2}+x_{3} \mathbf{e}_{3}=y_{1} \mathbf{e}_{1}+y_{2} \mathbf{e}_{2}+x_{3} \boldsymbol{\alpha}$.

\subsection{Construction of non-naive arithmetic discrete planes}

I. Debled-Rennesson has given several algorithms [Deb95] computing the geometric representation of rational naive discrete planes, which rely on functionality of such planes. Generalized functionality enables us to adapt these algorithms to any rational arithmetic discrete plane having a functional vector $\boldsymbol{\alpha} \in \mathbb{Z}^{3}$ satisfying $\alpha_{3}=1$. For the sake of clarity, we have chosen to work out in full details only one algorithm. The other ones can be extended similarly. 
Lemma 30 is the key point of the generation algorithm given below (see Algorithm 1). Indeed, instead of constructing the arithmetic discrete plane $\mathcal{P}$ in $\mathbb{Z}^{3}$ endowed with the basis $\left\{\mathbf{e}_{\mathbf{1}}, \mathbf{e}_{\mathbf{2}}, \mathbf{e}_{\mathbf{3}}\right\}$, we compute the points of $\mathcal{P}$ as the ones of a naive arithmetic discrete plane related to the basis $\left\{\mathbf{e}_{\mathbf{1}}, \mathbf{e}_{\mathbf{2}}, \boldsymbol{\alpha}\right\}$ (see Lemma 30). We finally reconstruct $\mathcal{P}$ by to the change of basis $\left\{\mathbf{e}_{\mathbf{1}}, \mathbf{e}_{\mathbf{2}}, \boldsymbol{\alpha}\right\}$ to $\left\{\mathbf{e}_{1}, \mathbf{e}_{2}, \mathbf{e}_{3}\right\}$.

A first idea for constructing the geometric representation of $\mathcal{P}$ consists in computing the value $H_{\mathcal{P}, \boldsymbol{\alpha}}(\mathbf{y})$ for each $\mathbf{y} \in \Gamma_{\boldsymbol{\alpha}}$. Nevertheless, the integer division makes this method relatively slow. We thus introduce the map

$$
R_{\mathcal{P}, \boldsymbol{\alpha}}: \Gamma_{\boldsymbol{\alpha}} \longrightarrow \mathbb{Z}, \mathbf{y}=y_{1} \mathbf{e}_{\mathbf{1}}+y_{2} \mathbf{e}_{\mathbf{2}} \longmapsto v_{1} y_{1}+v_{2} y_{2}+w H_{\mathcal{P}, \boldsymbol{\alpha}}(\mathbf{y})+\mu .
$$

An easy computation gives:

Lemma 31 Let $\mathcal{P}=\mathfrak{P}(\mathbf{v}, \mu, w)$ be a rational arithmetic discrete plane. Let $\boldsymbol{\alpha} \in \mathbb{Z}^{3}$ be a functional vector of $\mathcal{P}$ with $\alpha_{3}=1$ and let $H_{\mathcal{P}, \boldsymbol{\alpha}}: \mathbb{Z}^{2} \longrightarrow \mathbb{Z}$ be the height function of $\mathcal{P}$ related to the functional vector $\boldsymbol{\alpha}$. Then:

$$
\begin{aligned}
& H_{\mathcal{P}, \boldsymbol{\alpha}}\left(\mathbf{y}+\mathbf{e}_{\mathbf{i}}\right)-H_{\mathcal{P}, \boldsymbol{\alpha}}(\mathbf{y})= \begin{cases}-\left\lfloor v_{i} / w\right\rfloor & \text { if } R_{\mathcal{P}, \boldsymbol{\alpha}}(\mathbf{y})+\overline{v_{i}}<w \\
-\left\lfloor v_{i} / w\right\rfloor-1 & \text { otherwise }\end{cases} \\
& R_{\mathcal{P}, \boldsymbol{\alpha}}\left(\mathbf{y}+\mathbf{e}_{\mathbf{i}}\right)-R_{\mathcal{P}, \boldsymbol{\alpha}}(\mathbf{y})= \begin{cases}\overline{v_{i}} & \text { if } R_{\mathcal{P}, \boldsymbol{\alpha}}(\mathbf{y})+\overline{v_{i}}<w \\
\overline{v_{i}}-w & \text { otherwise }\end{cases}
\end{aligned}
$$

where $\overline{v_{i}}=v_{i} \bmod w$.

Hence, given the rectangle $\llbracket m_{1}, m_{1}^{\prime} \rrbracket \times \llbracket m_{2}, m_{2}^{\prime} \rrbracket$, one constructs the pre-images of the points $\mathbf{y}=y_{1} \mathbf{e}_{\mathbf{1}}+y_{2} \mathbf{e}_{\mathbf{2}} \in \Gamma_{\boldsymbol{\alpha}}$, with $\left(y_{1}, y_{2}\right) \in \llbracket m_{1}, m_{1}^{\prime} \rrbracket \times \llbracket m_{2}, m_{2}^{\prime} \rrbracket$ by first computing the height $\mathrm{H}\left(\mathbf{y}_{\mathbf{0}}\right)$ of the point $\mathbf{y}_{\mathbf{0}}=m_{1} \mathbf{e}_{\mathbf{1}}+m_{2} \mathbf{e}_{\mathbf{2}} \in \Gamma_{\boldsymbol{\alpha}}$, and, then, step by step, and thanks to Lemma 31 , by calculating the height $\mathrm{H}(\mathbf{y})$ of each point $\mathbf{y}=y_{1} \mathbf{e}_{\mathbf{1}}+y_{2} \mathbf{e}_{\mathbf{2}} \in \Gamma_{\boldsymbol{\alpha}}$, where $\left(y_{1}, y_{2}\right) \in \llbracket m_{1}, m_{1}^{\prime} \rrbracket \times \llbracket m_{2}, m_{2}^{\prime} \rrbracket$ (see Algorithm 1).

\subsection{Arithmetic discrete planes as combinatorial manifolds}

Due to the surface nature of Euclidean planes, it becomes natural to try to endow rational arithmetic discrete planes with a relevant notion of discrete surface. The notion of discrete surface has been widely studied during the last 25 years and several apporaches have been chosen for the definition of such objects. For instance, in [MR81], D. G. Morgenthaler and A. Rosenfeld define a discrete surface as a 0 -connected subset of $\mathbb{Z}^{3}$ satisfying some local conditions of 0 -adjacency and 2-separatingness. Nevertheless, this definition 
$\overline{\text { Algorithm 1: An algorithm constructing the arithmetic plane } \mathfrak{P}(\mathbf{v}, \mu, w)}$ satisfying $\mathbf{v} \in \mathbb{N}^{3}, \mu \in \mathbb{Z}$ and $w=\alpha_{1} v_{1}+\alpha_{2} v_{2}+v_{3}$ with $\left(\alpha_{1}, \alpha_{2}\right) \in \mathbb{Z}^{2}$.

Input: a rational arithmetic discrete plane $\mathcal{P}=\mathfrak{P}(\mathbf{v}, \mu, w)$ with $w \geq\left\{v_{1}, v_{2}\right\}$;

a functional vector $\boldsymbol{\alpha} \in \mathbb{Z}^{3} \mathcal{P}$ with $\alpha_{3}=1$;

the ranges $\left[m_{0}, m_{1}\right] \times\left[n_{0}, n_{1}\right]$;

Output: the points of $\mathcal{P}=\mathfrak{P}(\mathbf{v}, \mu, w)$ with $x$-coordinates in $\left[m_{0}, m_{1}\right]$ and $y$-coordinnates in $\left[n_{0}, n_{1}\right]$ in the basis $\left\{\mathbf{e}_{\mathbf{1}}, \mathbf{e}_{\mathbf{2}}, \boldsymbol{\alpha}\right\}$ of $\mathbb{Z}^{3}$.

$$
\begin{array}{rlrl}
\mathrm{z}_{\mathrm{ini}} & =-\left\lfloor\frac{\mathrm{v}_{1} \mathrm{~m}_{0}+\mathrm{v}_{2} \mathrm{n}_{0}+\mu}{\mathrm{w}}\right\rfloor ; & \mathrm{z} & =\mathrm{z}_{\mathrm{ini}} ; \\
\mathrm{x}_{\mathrm{ini}} & =\mathrm{m}_{0}+\alpha_{1} \mathrm{z} ; & \mathrm{x} & =\mathrm{x}_{\mathrm{ini}} ; \\
\mathrm{y}_{\mathrm{ini}} & =\mathrm{n}_{0}+\alpha_{2} \mathrm{z} ; & \mathrm{y}=\mathrm{y}_{\mathrm{ini}} ; \\
\mathrm{r}_{\mathrm{ini}} & =\mathrm{v}_{1} \mathrm{~m}_{0}+\mathrm{v}_{2} \mathrm{n}_{0}+\mathrm{wz}_{\mathrm{ini}}+\mu ; & \mathrm{r} & =\mathrm{r}_{\mathrm{ini}} ;
\end{array}
$$

\section{begin}

$$
\text { for } \mathrm{n} \in\left\{\mathrm{n}_{0}, \ldots, \mathrm{n}_{1}\right\} \text { do }
$$

for $\mathrm{m} \in\left\{\mathrm{m}_{0}, \ldots, \mathrm{m}_{1}\right\}$ do

Build the voxel $(\mathrm{x}, \mathrm{y}, \mathrm{z})$;

$r=r+\overline{v_{1}} ; \quad x=x+1$;

if $r<w$ then

$\mid \mathrm{z}=\mathrm{z}-\left\lfloor\frac{\mathrm{v}_{1}}{\mathrm{w}}\right\rfloor ; \mathrm{x}=\mathrm{x}-\alpha_{1}\left\lfloor\frac{\mathrm{v}_{1}}{\mathrm{w}}\right\rfloor ; \mathrm{y}=\mathrm{y}-\alpha_{2}\left\lfloor\frac{\mathrm{v}_{1}}{\mathrm{w}}\right\rfloor ;$

else

$\mathrm{z}=\mathrm{z}-\left(\left\lfloor\frac{\mathrm{v}_{1}}{\mathrm{w}}\right\rfloor+1\right) ; \mathrm{x}=\mathrm{x}-\alpha_{1}\left(\left\lfloor\frac{\mathrm{v}_{1}}{\mathrm{w}}\right\rfloor+1\right) ;$

$$
\mathrm{y}=\mathrm{y}-\alpha_{2}\left(\left\lfloor\frac{\mathrm{v}_{1}}{\mathrm{w}}\right\rfloor+1\right) \text {; }
$$

\section{end}

end

$\mathrm{r}_{\mathrm{ini}}=\mathrm{r}_{\mathrm{ini}}+\overline{\mathrm{v}_{2}} ; \mathrm{y}_{\mathrm{ini}}=\mathrm{y}_{\mathrm{ini}}+1$;

if $r_{\text {ini }}<w$ then

$$
\mathrm{z}_{\mathrm{ini}}=\mathrm{z}_{\mathrm{ini}}-\left\lfloor\frac{\mathrm{v}_{2}}{\mathrm{w}}\right\rfloor ; \mathrm{x}_{\mathrm{ini}}=\mathrm{x}_{\mathrm{ini}}-\alpha_{1}\left\lfloor\frac{\mathrm{v}_{2}}{\mathrm{w}}\right\rfloor ; \mathrm{y}_{\mathrm{ini}}=\mathrm{y}_{\mathrm{ini}}-\alpha_{2}\left\lfloor\frac{\mathrm{v}_{2}}{\mathrm{w}}\right\rfloor ;
$$

else

$$
\begin{aligned}
& \mathrm{z}_{\mathrm{ini}}=\mathrm{z}_{\mathrm{ini}}-\left(\left\lfloor\frac{\mathrm{v}_{2}}{\mathrm{w}}\right\rfloor+1\right) ; \\
& \mathrm{x}_{\mathrm{ini}}=\mathrm{x}_{\mathrm{ini}}-\alpha_{1}\left(\left\lfloor\frac{\mathrm{v}_{2}}{\mathrm{w}}\right\rfloor+1\right) ; \\
& \mathrm{y}_{\mathrm{ini}}=\mathrm{y}_{\mathrm{ini}}-\alpha_{2}\left(\left\lfloor\frac{\mathrm{v}_{2}}{\mathrm{w}}\right\rfloor+1\right) ;
\end{aligned}
$$

\section{end}

$\mathrm{x}=\mathrm{x}_{\mathrm{ini}} ; \mathrm{y}=\mathrm{y}_{\mathrm{ini}} ; \mathrm{z}=\mathrm{z}_{\mathrm{ini}} ; \mathrm{r}=\mathrm{r}_{\mathrm{ini}}$

end 
is not relevant for rational arithmetic discrete planes. For instance, among the 40 tricubes occurring in the naive arithmetic discrete planes with positive normal vector $\mathbf{v} \in \mathbb{Z}^{3}$, such that $0 \leq v_{1} \leq v_{2} \leq v_{3}$, only 7 of them occur in a discrete surface in the sense of D. G. Morgenthaler and A. Rosenfeld [MB99].

In [Fra96,KI00,KI03], the authors have shown that an appropriate way to provide rational arithmetic discrete planes with a discrete surface structure is to consider two-dimensional combinatorial manifolds. For instance, J. Françon showed in [Fra96] that the 2-adjacency graph of a rational standard arithmetic discrete plane has a natural underlying structure of two-dimensional combinatorial manifold. In [KI00], Y. Kenmochi and A. Imiya, thanks to a similar approach, proved that one can provide any rational naive arithmetic discrete plane $\mathcal{P}$ with two different structures of two-dimensional combinatorial manifolds, depending on the 0 -adjacency and the 1 -adjacency graph of $\mathcal{P}$ respectively (see Figure 6).

In the present section, we show that these latter approaches can be extended in a quite natural way to any rational arithmetic discrete plane $\mathcal{P}$ with normal vector $\mathbf{v} \in \mathbb{Z}^{3}$ under the assumption $w-v_{3} \in \operatorname{gcd}\left(v_{1}, v_{2}\right) \mathbb{Z}$ and $w \geq$ $\max \left\{v_{1}, v_{2}\right\}$. We have chosen to consider 1-adjacency only, 0-adjacency being handled in a similar way.

Let us first recall basic notions concerning combinatorial manifolds. Let $G$ be a graph and let $F$ be a set of elementary cycles of $G$, that is, circular permutations $\left(V_{1}, V_{2}, \ldots, V_{k}\right)$, with $k>2$, of vertices of $G$ such that $V_{i} \neq V_{j}$ if $i \neq j$ and, for $i \in \mathbb{N}$, any pair $\left\{V_{i}, V_{i+1}\right\}$ is an edge of $G$ (the indices are considered modulo $k$ ). Such a cycle is called a face. Two faces are called adjacent if they share an edge. An umbrella at a vertex $V$ of $G$ is a circular permutation $\left(F_{1}, F_{2}, \ldots, F_{k}\right)$ of faces of $F$, with $k>1$, all sharing a vertex $V$, and such that $F_{i}$ and $F_{i+1}$ are adjacent for $i \in \mathbb{N}$ (indices are considered modulo $k$ ).

Definition 32 Let $G$ be a graph and let $F$ be a set of faces of $G$. The pair $(G, F)$ is called a two-dimensional combinatorial manifold (without boundary) if the following holds:

(i) any edge of $G$ belongs to exactly two faces of $G$;

(ii) any vertex of $G$ belongs to exactly one umbrella of $G$.

Since rational naive arithmetic discrete planes can be provided with a twodimensional combinatorial manifold structure [KIO0], then, thanks to Lemma 30 , there is a quite natural way to provide any rational arithmetic discrete plane $\mathfrak{P}(\mathbf{v}, \mu, w)$ having a functional vector $\boldsymbol{\alpha} \in \mathbb{Z}^{3}$ satisfying $\alpha_{3}=1$. According to Lemma 30 , the arithmetic discrete plane $\mathfrak{P}(\mathbf{v}, \mu, w)$ is naive relatively to the basis $\left\{\right.$ vecte $\left._{1}, \mathbf{e}_{\mathbf{2}}, \boldsymbol{\alpha}\right\}$ and can be provided with a two-dimensional com- 

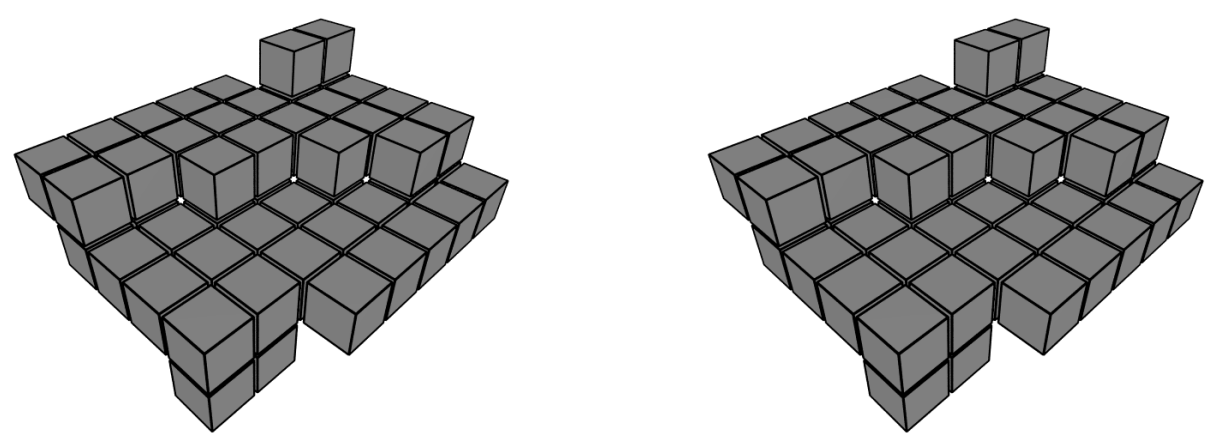

(a) The arithmetic discrete plane $0 \leq$ (b) The arithmetic discrete plane $0 \leq$ $6 x_{1}+11 x_{2}+31 x_{3}<42$ represented by $6 x_{1}+11 x_{2}+42 x_{3}<42$ represented by voxels. voxels.
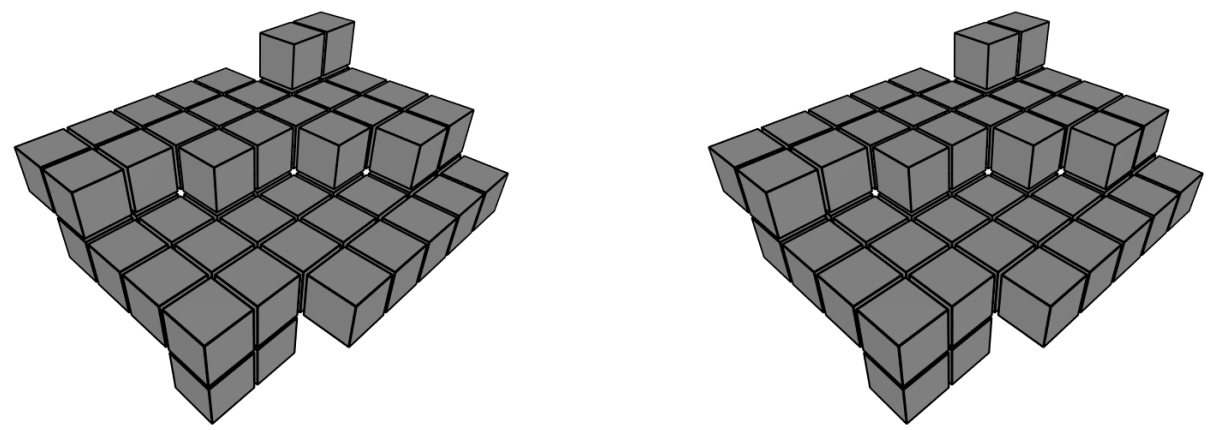

(c) The two-dimensional combinatorial (d) The two-dimensional combinatorial manifold $\mathfrak{M}((6,11,31), 0,42)$. manifold $\mathfrak{M}((6,11,42), 0,42)$.

Fig. 6. From a thick arithmetic discrete plane to a two-dimensional combinatorial manifold .

binatorial manifold structure. Let us introduce the bijection map

$$
\begin{aligned}
\phi: \mathfrak{P}(\mathbf{v}, \mu, w) & \longrightarrow \mathfrak{P}\left(\left(v_{1}, v_{2}, w\right), 0, w\right) \\
\left(x_{1}, x_{2}, x_{3}\right) & \longmapsto\left(x_{1}-\alpha_{1} x_{3}, x_{2}-\alpha_{2} x_{3}, x_{3}\right) .
\end{aligned}
$$

By $\phi$, the arithmetic discrete planes $\mathfrak{P}(\mathbf{v}, \mu, w)$ and $\mathfrak{P}\left(\left(v_{1}, v_{2}, w\right), 0, w\right)$ are identified. We thus define a two-dimensional combinatorial manifold $\mathfrak{M}(\mathbf{v}, \mu, w)$ over $\mathfrak{P}(\mathbf{v}, \mu, w)$ as follows: the vertices of $\mathfrak{M}(\mathbf{v}, \mu, w)$ are the points of $\mathfrak{P}(\mathbf{v}, \mu, w)$ and for all $\left(\mathbf{x}, \mathbf{x}^{\prime}\right) \in \mathfrak{P}(\mathbf{v}, \mu, w)^{2},\{\mathbf{x}, \mathbf{y}\}$ is an edge if, and only if, $\phi(\mathbf{x})$ and $\phi(\mathbf{y})$ are 1-adjacent in $\mathfrak{P}\left(\left(v_{1}, v_{2}, w\right), \mu, w\right)$. Finally, the faces of $\mathfrak{M}(\mathbf{v}, \mu, w)$ are the image by $\phi$ of the faces of the naive arithmetic discrete plane $\mathfrak{P}\left(\left(v_{1}, v_{2}, w\right), \mu, w\right)$ (see Figure 6). 


\section{Conclusion}

The aim of the present work was to introduce suitable tools in order to generalize well-known properties of naive arithmetic discrete planes. We have exhibited a generalized functionality for arithmetic discrete planes $\mathcal{P}=\mathfrak{P}(\mathbf{v}, \mu, \omega)$ by introducing a suitable projection direction $\boldsymbol{\alpha}$. We have proved that, as soon as $|\langle\boldsymbol{\alpha}, \mathbf{v})\rangle \mid=w$ and $\operatorname{gcd}\left(\alpha_{1}, \alpha_{2}, \alpha_{3}\right)=1$, there is a one-to-one correspondence between $\mathcal{P}$ and a two-dimensional lattice $\Gamma_{\boldsymbol{\alpha}}$; we also have described the latter lattice. We then have focused on the class of arithmetic discrete planes for which there exists $\boldsymbol{\alpha}$ with $\alpha_{3}=1$ : we first have investigated plane partitions and local configurations; indeed we have extended the well-known result on the number of $(m, n)$-configurations in a naive plane (there are at most $m n$ such configurations), and we have considered their statistical behaviour; we finally have proved that arithmetic discrete planes with $\alpha_{3}=1$ and $w \geq \max \left\{v_{1}, v_{2}\right\}$ can be considered as naive in a suitable basis of $\mathbb{R}^{3}$; we have deduced a generation algorithm and shown how to endow them with a structure of two-dimensional manifold.

The approach developed in Section 5 is classical in symbolic dynamics and, in particular, in the study of sturmian and multidimensional sturmian words [PF02]. As an analogous example of application of symbolic dynamics in discrete geometry see also [BN05].

The results of the present paper offer new perspectives for further investigation of general properties of arithmetic discrete planes of any thickness. In particular, we plan to use them to generate arbitrarily large parts of discrete planes via symbolic substitutions following [ABS04], to recover the corresponding Farey tesselation as well as the symmetry properties of $(m, n)$-cubes of a discrete plane [VC99], and finally as a new approach to the recognition problem of discrete planes [FST96,FP99,VC00].

\section{References}

[AAS97] É. Andres, R. Acharya, and C. Sibata. The Discrete Analytical Hyperplanes. Graph. Models Image Process., 59(5):302-309, 1997.

[ABS04] P. Arnoux, V. Berthé, and A. Siegel. Two-dimensional Iterated Morphisms and Discrete Planes. Theoret. Comput. Sci., 319:145-176, 2004.

[BBN00a] R.P. Barneva, V.E. Brimkov, and P. Nehlig. Thin discrete triangular meshes. Theoret. Comput. Sci. 246(1-2):73-105, 2000.

[BN05] V. Berthé and B. Nouvel. Density of symbols in discretized rotation configurations. In Words 2005 - 5th International Conference on Words, 
volume 36 of Publications du LaCIM, Université du Québec à Montréal, pages 163-173. Universit du Québec Montréal, September 2005.

[BV00] V. Berthé and L. Vuillon. Tilings and Rotations on the Torus: A Two-Dimensional Generalization of Sturmian Sequences. Discrete Math., $223: 27-53,2000$.

[BV01] V. Berthé and L. Vuillon. Palindromes and Two-Dimensional Sturmian Sequences. J. Autom. Lang. Comb., 6(2):121-138, 2001.

[BFJ05] V. Berthé, C. Fiorio, and D. Jamet. Generalized functionality for arithmetic discrete planes. In DGCI 2005, 12th International Conference, volume 3429 of Lecture Notes in Computer Science, pages 276-286, Springer, 2005.

[BAB02] V. E. Brimkov, E. Andres, and R. P. Barneva. Object discretizations in higher dimensions. Pattern Recogn. Let., 23 (6): 623-636, 2002.

[BB99] V. E. Brimkov and R. P. Barneva. Graceful Planes and Thin Tunnel-Free Meshes. In DGCI, 8th International Conference, volume 1568 of LNCS, pages 53-64, 1999.

[BBN00b] V. E. Brimkov, R. P. Barneva, and P. Nehlig. Minimally thin discrete triangulations. In Volume Graphics, A. Kaufman, R. Yagel, M. Chen (Eds.), Chapter 3, pages 51-70, Springer, 2000.

[BB02] V. E. Brimkov and R. P. Barneva. Graceful Planes and Lines. Theoret. Comput. Sci., 283:151-170, 2002.

[BK04] V. E. Brimkov, R. Klette. Curves, hypersurfaces, and good pairs of adjacency relations. Lecture Notes in Computer Science, No 3322:270284, Springer, 2004.

[Col02] M. A. Jacob-Da Col. About Local Configurations in Arithmetic Planes. Theoret. Comput. Sci., 283:183-201, 2002.

[DRR03] I. Debled-Rennesson, J.-L. Rémy, and J. Rouyer-Degli. Segmentation of discrete curves into fuzzy segments. In 9th International Workshop on Combinatorial Image Analysis - Electronic Notes in Discrete Mathematics, volume 12, page 12, Palermo, Italy, May 14-16 2003. Elsevier.

[Deb95] I. Debled-Renesson. Reconnaissance des Droites et Plans Discrets. Thèse de doctorat, Université Louis Pasteur, Strasbourg, France, 1995.

[DR94] I. Debled-Renesson and J.-P. Reveillès. A New Approach to Digital Planes. In Vision geometry III, Proc. SPIE, volume 2356, Boston, USA, 1994.

[Fra96] J. Françon. Sur la topologie d'un plan arithmétique. Theoret. Comput. Sci., 156(1-2):159-176, 1996.

[FP99] J. Françon and L. Papier. Polyhedrization of the boundary of a voxel object. In DGCI, 8th International Conference, volume 1568 of LNCS, pages 425-434, 1999. 
[FST96] J. Françon, J.-M. Schramm, and M. Tajine. Recognizing Arithmetic Straight Lines and Planes. In DGCI, 6th International Workshop, LNCS, pages 141-150, 1996.

[Gér99] Y. Gérard. Local Configurations of Digital Hyperplanes. In DGCI, 8th International Conference, volume 1568, pages 65-75, 1999.

[KI00] Y. Kenmochi and A. Imiya. Naive planes and discrete combinatorial surfaces. In Proceedings of the 9th International Conference on Discrete Geometry for Computer Imagery, pages 249-261. Springer-Verlag, 2000.

[KI03] Y. Kenmochi and A. Imiya. Combinatorial topologies for discrete planes. In DGCI, 11th International Conference, DGCI 2003, volume 2886 of Lecture Notes in Computer Science, pages 144-153, Springer-Verlag, 2003.

[KN85] L. Kuipers and H. Niederreiter. Uniform distribution of sequences. (Ravnomernoe raspredelenie posledovatel'nostej). Transl. from the English. Transl. ed. by S. M. Ermakov. Moskva: Nauka. Glavnaya Redaktsiya Fiziko-Matematicheskoj Literatury. 408 p. , 1985.

[MB99] R. Malgouyres and G. Bertrand. Complete local characterization of strong 26-surfaces : Continuous analogs for strong 26-surfaces. International Journal on Pattern Recognition and Artificial Intelligence (IJPRAI), 13(4):465-484, 1999.

[MR81] D. G. Morgenthaler and A. Rosenfeld. Surfaces in three-dimensional digital images. Information and Control, 51:227-247, 1981.

[PF02] N. Pytheas Fogg. Substitutions in dynamics, arithmetics and combinatorics. Springer Verlag, Lecture notes in Mathematics, volume 1794, 2002, Eds. V. Berth, S. Ferenczi, C. Mauduit, A. Siegel.

[Rev91] J.-P. Reveillès. Calcul en Nombres Entiers et Algorithmique. Thèse d'état, Université Louis Pasteur, Strasbourg, France, 1991.

[Rev95] J.-P. Reveillès. Combinatorial Pieces in Digital Lines and Planes. In Vision geometry IV, Proc. SPIE, 2573, volume 2573, pages 23-24, San Diego, CA, 1995 .

[Rot94] G. Rote. Sequences with subword complexity 2n. J. Number Theory, 46:196-213, 1994.

[Sch97] J.-M. Schramm. Coplanar Tricubes. In DGCI, 7th International Workshop, volume 1347 of LNCS, pages 87-98, 1997.

[VC97] J. Vittone and J.-M. Chassery. Coexistence of Tricubes in Digital Naive Plane. In DGCI, rth International Workshop, volume 1347 of LNCS, pages 99-110, 1997.

[VC99] J. Vittone and J.-M. Chassery. (n,m)-cubes and Farey Nets for Naive Planes Understanding. In DGCI, 8th International Conference, volume 1568 of LNCS, pages 76-87, Springer-Verlag, 1999. 
[VC00] J. Vittone and J.-M. Chassery. Recognition of Digital Naive Planes and Polyhedrization. In DGCI, 9th International Conference, volume 1953 of LNCS, pages 296-307, Springer-Verlag, 2000.

[Vui99] L. Vuillon. Local Configurations in a Discrete Plane. Bull. Belgian Math. Soc., 6:625-636, 1999. 